\title{
Multi-functionalization Strategies Using Nanomaterials: A Review and Case Study in Sensing Applications
}

\author{
$\mathrm{Ji}$-Hyeon Song ${ }^{1} \cdot$ Soo-Hong $\mathrm{Min}^{2} \cdot$ Seung-Gi Kim ${ }^{2} \cdot$ Younggyun $\mathrm{Cho}^{2} \cdot$ Sung-Hoon $\mathrm{Ahn}^{2,3}$ (i)
}

Received: 23 January 2021 / Revised: 6 April 2021 / Accepted: 14 April 2021 / Published online: 10 May 2021

(c) The Author(s) 2021

\begin{abstract}
Remarkable advances in nanomaterials and nanotechnology have led researchers in various fields. The scale effects imparted by nanomaterials are associated with unexpected macroscale phenomena and properties that find many applications. However, multi-functionalization may be accompanied by physical and commercial limitations. Therefore, research must proceed in several different directions. Here, we define multi-functionalization and the electrical applications thereof in terms of increasing performance, addition of new and valuable properties, and multi-physics in play. We deal with sensors, actuators, energy harvesters, and solar cells and explore research that seeks to increase sensitivity, append "stretchability", and facilitate untethered communication. Furthermore, we analyze research trends in materials use and manufacturing, and highlight useful fabrication methods. With the aim of predicting future research trends, our review presents a roadmap that will aid research on sensing and multi-functional applications.
\end{abstract}

Keywords Nanomaterials $\cdot$ High performance $\cdot$ Multi-properties $\cdot$ Multi-physics $\cdot$ Sensing applications

\section{Introduction}

Applications of nanotechnology have received a great deal of attention since the late 1980s. In the United States, for example, federal government funding in nanotechnology increased by $15 \%$ annually from 2001 to 2010 , and stands at over $\$ 1.6$ billion today [1]. Large companies such as GE and Intel devote significant resources to nanotechnology [2, 3]. Carbon nanotubes (CNTs) and graphene lattices created from nanoconfined forms of graphite have been used to create high-performance transistors and ultra-strong composite materials $[4,5]$. These materials have been employed to

Ji-Hyeon Song and Soo-Hong Min equally contributed to this work.

Invited Review: This paper is an invited paper.

Sung-Hoon Ahn

ahnsh@snu.ac.kr

1 Department of Mechanical Engineering, Dankook University, Yongin, Gyeonggi-do 16890, Republic of Korea

2 Department of Mechanical Engineering, Seoul National University, Seoul 08826, Republic of Korea

3 Institute of Advanced Machines and Design, Seoul National University, Seoul 08826, Republic of Korea manufacture new displays, super-efficient batteries, and solar cells [6-8]. Pharmaceutical companies seek to use nanoparticles to target drugs for diseases such as cancer $[9,10]$. In the fields of electronics and semiconductor manufacturing, graphene-based electronics, spintronics, and photonics may replace silicon [11-13]. Nano-structuring increases the battery storage capacity, yields cheap and efficient solar cells, and delivers ultra-strong composites [13-15]. Nanomaterials are used to produce smart materials that are self-healing or self-cleaning, memory metals that revert to their original shapes, and piezoelectric ceramics and crystals that turn pressure into energy [16-19].

Here, we review nanomaterials ranging from several tens of nanometers to several micrometers in size (Fig. 1); thus, nanoplates, nanoparticles, nanowires, and nanotubes find most applications [20]. Their behavior is governed by both quantum and classical physics [21]. For example, nanomaterials have much greater surface areas than macro-scale materials of similar weights. Furthermore, nanomaterials interact very extensively with their surroundings [22].

Ordinary substances have different properties on the nanoscale; thus, greater reactivity, unusual electrical behavior, and remarkable strength per weight allows the creation of new medicines, super-slick coatings, and stronger composites [23-25]. Figure 2 shows that nanomaterials exhibit 


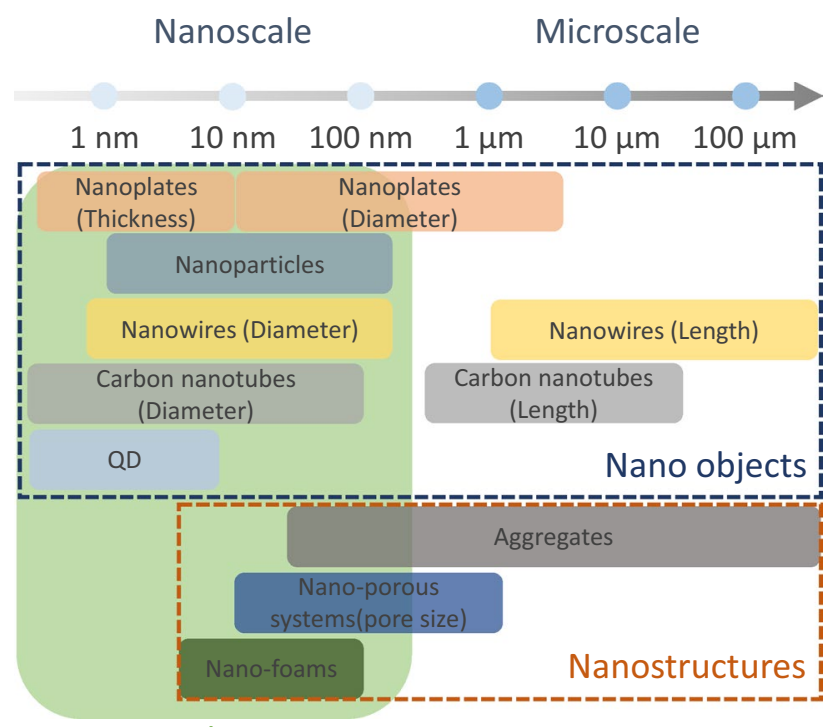

Scope in this review

Fig. 1 The scales of nanomaterials with respect to nano objects and nanostructures

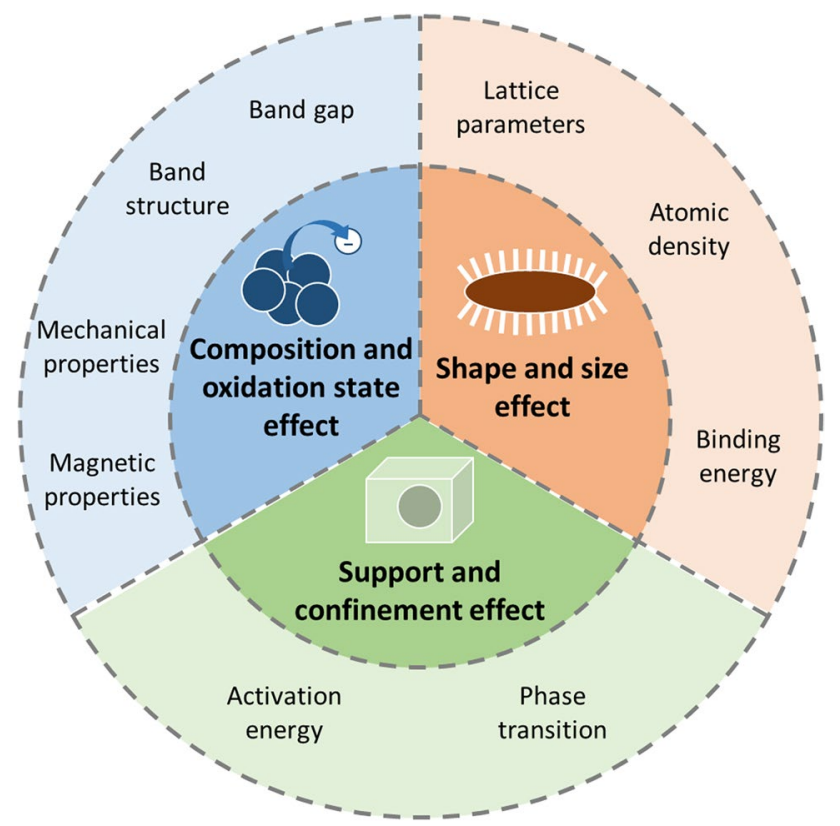

Fig. 2 The physical attributes of various nanomaterials with respect to composition and oxidation effect, shape and size effect, and support and confinement effect

(1) support and confinement effects, (2) shape and size effects, and (3) composition and oxidation state effects. The first effect occurs when the nanomaterial scale is comparable to the wavelength of an electron [26]. The motion of randomly moving electrons is restricted to specific energy levels, and the quantum realm reigns [27]. Such effects depend on the material properties, Bohr radius, available energy levels, potential well, and valence and conduction bands [28].

Nanomaterial shape and size (the "morphology effect") influence the melting point, fluorescence, electrical conductivity, magnetic permeability, and chemical reactivity of the materials [29-33]. Vacancies ("missing" atoms in a lattice) play major roles in electrical and thermal transport and mechanical behavior [34, 35]. Furthermore, nanomaterials may exhibit a variety of oxidation states and are easily oxidized by ambient air (which is sometimes undesirable) [36]. Usually, the composition and oxidation status of magnetic nanomaterials are determined by the chosen synthetic phase, which may include both surfactants and precursor ligands [37, 38].

The explosive interest in nanomaterials is reflected in the number of papers listed in the Science Citation Index (Extended) (SCI(E)). We analyzed research trends of 2011-2013, 2014-2016, and 2017-2019 based on the number of published articles and citations, impact factors, and the H-index [39, 40]. This index allows the analysis of journal quality and principal research trends. The $\mathrm{H}$-index evaluates the performance of individuals and the impact of their work. Figure 3 shows that research interest and quality increased greatly from 2011 to 2019 [41]. All nanotube, nanoparticle, quantum dot, nanowire, and nanoplate studies have increased greatly in number over the past five years. Carbon nanotubes (CNTs) are the most active topic in terms of the number of journal articles and the $\mathrm{H}$-index, followed by quantum dots and nanoparticles. Nanoplate research exhibited the most rapid increase in $\mathrm{H}$-index, and nanoparticles showed the slowest. Interest in nanomaterials shows no signs of abating.

Nanomaterials are used in a variety of products including paints, filters, insulators, and lubricants; their very small scales impart specific properties, and they have become invaluable tools in materials science [42-45]. Recently, nanomaterials have been applied in biosensing, bioimaging, tumor diagnosis, and anti-biofouling [46, 47]. Pharmaceutical companies use nano-enabled biotechnologies to produce rapid and sensitive diagnostic agents and more effective therapeutics [48]. The food manufacturing industry employs nanolaminates of edible lipids or polysaccharides to spray food products, protecting them from air and moisture [49]. Figure 4 shows the scope of nanomaterial applications, including sensors, actuators, energy harvesters and storage devices, solar cells, catalysts, healthcare materials, compounds used in food and agriculture, cosmetics and paint, and industries. In this study, we focus on their electrical applications. We will deal with sensors, actuators, energy harvesters, storage devices, and solar cells. Engineers seek to enhance performance (increase sensitivity), add new properties (for example, stretchability), ensure untethered communication, and develop a multi-physics that qualitatively 
Fig. 3 The H-index trajectory of keywords pertaining to nanomaterials used between 2011 and 2013, 2014-2016, and 2017-2019. Data were obtained from the Web of Science in December 2019

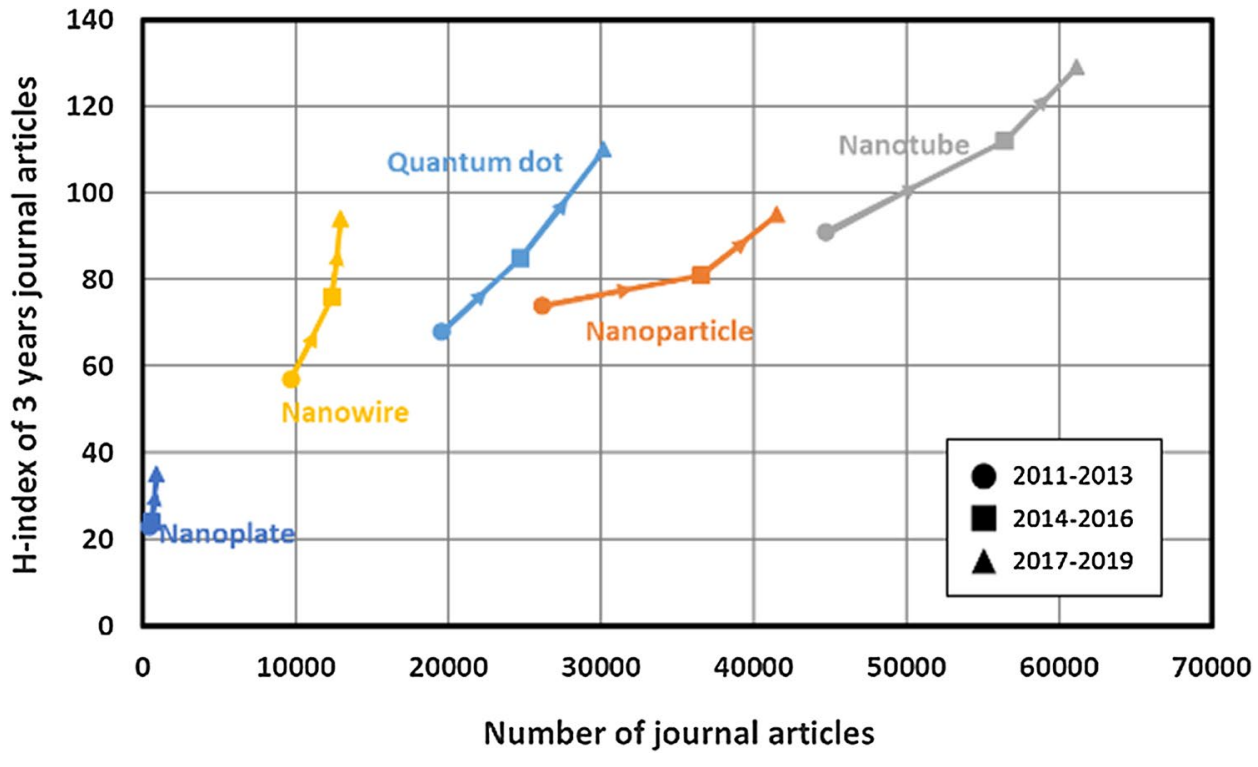

provide more than one attribute at a time, has been generated in the process of maximizing value. As new attributes have been added that have not been possible in a single product before, end-users could have benefited from using the product. It can be explained by a product bundling strategy that offers several products or services for sale as one combined product or service package in marketing [50,51]. A good bundle is worth more than the sum of its parts to the end user, replaces confusing or tedious choices with order and simplicity (Fig. 5). The extra properties afforded by nanomaterials generally reduce costs; even the convenience of not hand-picking and adding-on items is attractive. Since bundling of two different properties by nanomaterials can be effective in maximizing application value, researchers have been working on appending the properties of an existing product in various ways using nanomaterials.

\subsection{Physical Limitations of Nanomaterials}

The physical limitations of nanomaterials are usually evident during synthesis, which may be either top-down or bottom-up. During top-down synthesis, nanostructures are created by etching away existing crystal planes or by miniaturizing bulk fabrication [52]. Top-down processes dominate microscale fabrication but are limited by the wavelength of light and the available tools [53]. During bottom-up synthesis, nanostructures are synthesized on a substrate by atom stacking, which creates crystal planes [54]. The structures are smaller than those of top-down syntheses, but the surfaces and stacked molecules must be compatible [55]. Neither process yields completely homogeneous nanomaterials of the desired sizes and shapes (Fig. 6). 
Fig. 5 The added value afforded by nanomaterials (left) and maximization of multi-functionalization by bundling (right)

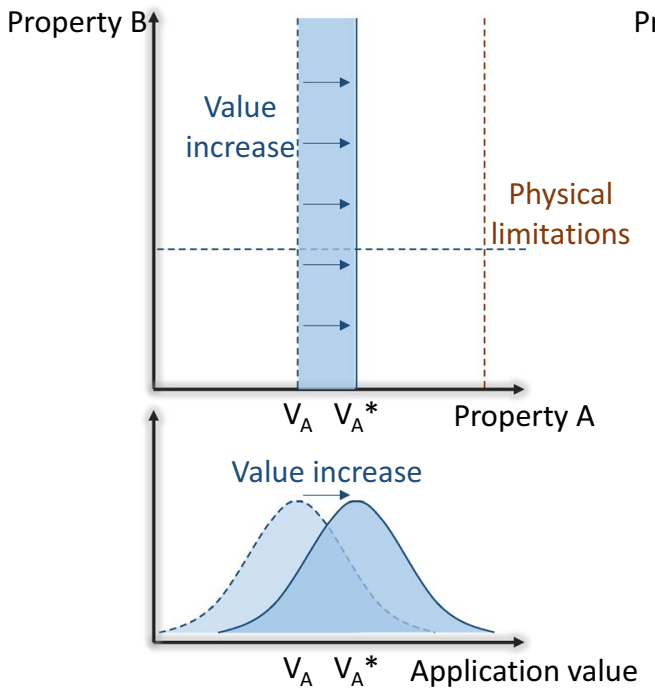

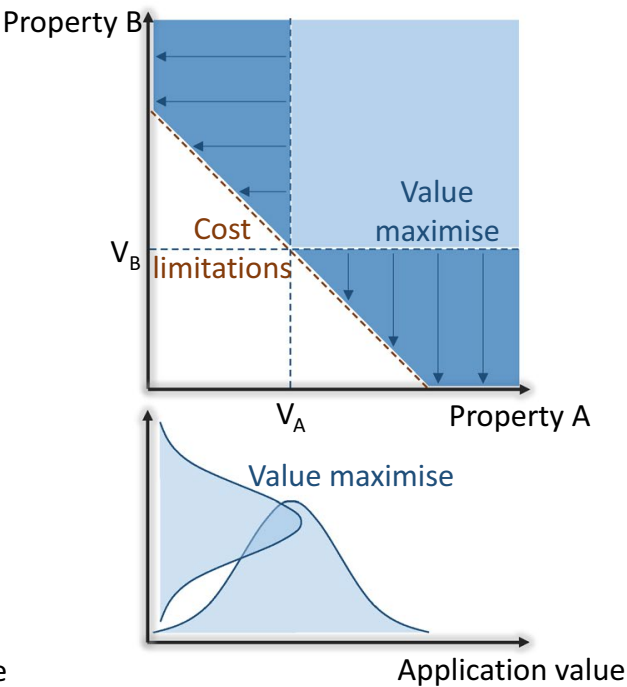

Free or surface energy imposes physical limitations during manufacturing. The surface (or Gibb's) energy is the difference in energy between a packed material and the same number of atoms in an infinitely extended solid [56, 57]. As nanomaterials exhibit large surfaceto-volume ratios (the "size effect"), their reactivities are very high [58]; thus, they exhibit large surface energies associated with a tendency to self-assemble and melt at low temperatures.

\subsection{Commercialization Limitations of Nanomaterials}

Nanomaterial commercialization is compromised by high processing costs, a lack of investment capital, poor process scalability, and a shortage of qualified engineers [59]. The investment timeline commences with a breakthrough invention, followed by attempted commercialization, and then (in successful cases) product acceptance and profit (Fig. 7; [60]). Researchers must bear in mind that expenditure is the greatest in the developmental phase and may be difficult to maintain.

\section{Multi-functionalize Strategy}

The term "multi-functionalization" is commonly used to explain new research trends, but confusion reigns: Does the term differ from "multi-physics" and "self-adjustability"? We define multi-functionalization as increasing performance, adding properties, and deriving multi-physics (Fig. 8). We sought trends in multi-functionalization; we do not focus on the historical phases of technological innovation, but are concerned with real-world applications. We

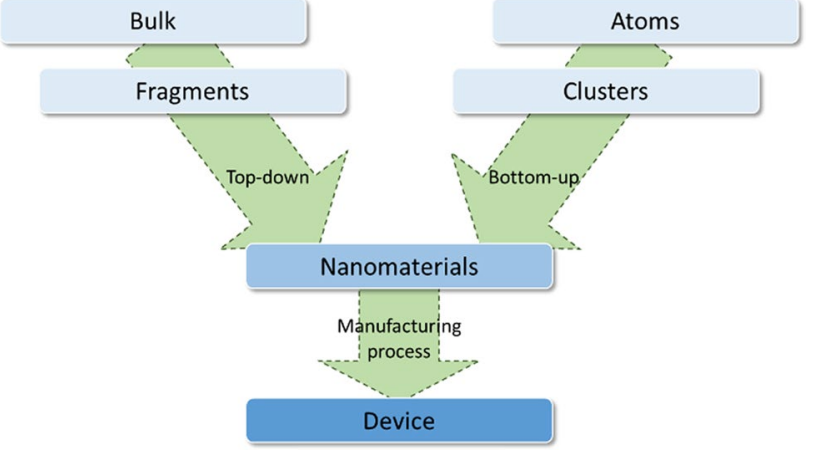

Fig. 6 The physical limitations of nanomaterials, from synthesis to inclusion in a device

seek to help researchers place their work in the context and suggest how they might best proceed. A performance increase reflects the value of a researcher's work and is appreciated by the end user. For example, enhanced sensor sensitivity and utility, a reduction in the actuating force, and an increase in the actuating frequency band are valued by end users. The novel properties of nanomaterials (Fig. 2) facilitate the addition of further properties, allowing (for example) construction of lightweight body armor, or clothes that resist wrinkling, staining, and bacterial growth. Clear nanoscale films on eyeglasses, computer and camera displays, windows, and other surfaces render them water- and residue-repellent, antireflective, self-cleaning, resistant to ultraviolet or infrared light, nonfogging, antimicrobially active, scratch-resistant, and electrically conductive. Nanomaterials are beginning to enable washable, durable "smart fabrics" equipped with flexible nanoscale sensors and electronics that monitor health and capture solar and motion energy. Furthermore, lightweighting of cars, trucks, airplanes, boats, and spacecraft saves significant fuel. Polymer 


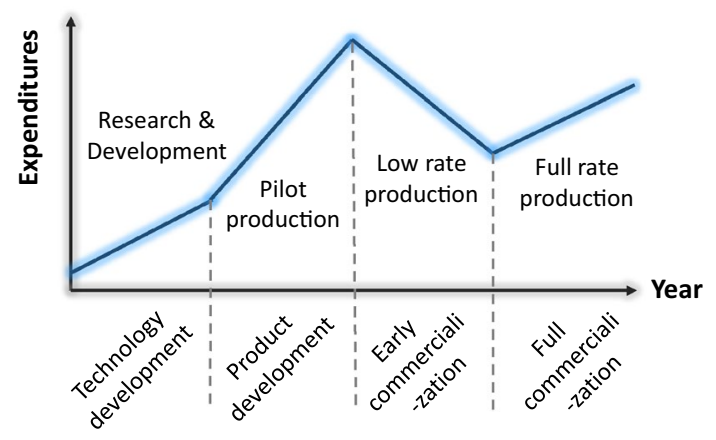

Fig. 7 The costs of commercial nanomaterials (modified from [60])

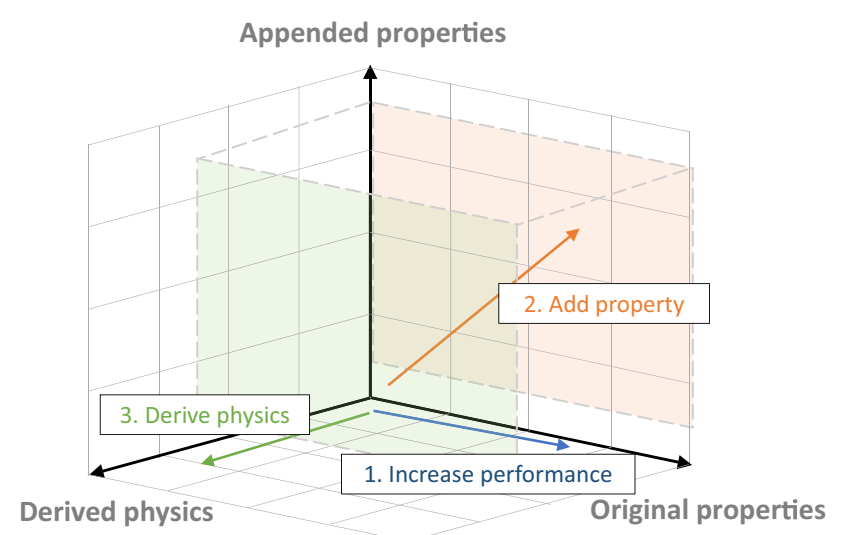

Fig. 8 Multi-functionalization increases performance, adds new properties, and derives new physics

composite nanomaterials are used to make golf clubs, tennis rackets, bicycles, motorcycle helmets, automobile parts, luggage, and power tool housings, all of which are lightweight, stiff, durable, and resilient. In addition, CNT sheets will soon be used in next-generation air vehicles.

Innovations may be incremental or radical. Performance

\begin{tabular}{|c|c|}
\hline Mechanical & Stiffness, strength, hardness, elongation at failure etc. \\
\hline Electrical & Electrical conductivity, resistivity, etc. \\
\hline Magnetic & Permittivity, permeability, etc. \\
\hline Optical & Reflectivity, transmissivity, absorptivity, emissivity, etc. \\
\hline Thermal & Heat capacity, thermal expansion, thermal conductivity, etc. \\
\hline Chemical & Toxicity, reactivity, flammability, oxidation state, etc. \\
\hline
\end{tabular}

Fig. 9 The possible properties afforded by nanomaterials

increases and property additions are usually incremental, and new physics is radical. Such physics reveals unsuspected applications and opens new markets. The research and development costs are high, as are the expected returns. The process is risky. The physics includes connections between mechanics, electromagnetics, and optics.

Of the three elements of multi-functionalization, the first is the improvement of properties, namely, mechanical, electrical, magnetic, optical, thermal, or chemical properties (Fig. 9). For example, the stiffness and strength are representative mechanical properties. The electrical properties include conductivity and resistivity. The second element is the addition of new properties, achieved using various nanomaterials and novel design strategies. The third element is new physics. Below, we will deal with sensors, actuators, energy harvesters, and solar cells. Thus, the original properties are electrical; as will become clear, most appended properties are mechanical. Some yield new magnetic, optical, thermal, or chemical physics.

\subsection{Sensors}

Nanotechnology has enabled electronic devices to become smaller and have a higher performance than before. Nanometer-scale materials and manufacturing processes quickly and effectively save and transmit data. Flexible, bendable, foldable, rollable, and stretchable electronics are being integrated into wearable medical applications, aerospace instruments, and the Internet of Things devices. Flexible electronics include semiconductor nanomembranes for smartphones and e-readers. Graphene and cellulosic nanomaterials are being used to create wearable tattoos, photovoltaics sewn into clothing, and rollable electronic paper. Flat, flexible, lightweight, non-brittle, highly efficient electronics find applications in a host of smart products. Other computing and electronic products include flash memory chips for smartphones and thumb drives, ultra-responsive hearing aids, antimicrobial/antibacterial keyboard coatings and cell phone casings, conductive inks for printed electronics [controlled via radio-frequency identification (RFID)], smart cards, smart packaging, and flexible e-book readers. Table 1 and Fig. 10 show the research trends and $\mathrm{H}$-index trajectories of the sensing applications. Sensor sensitivity is commonly used to evaluate sensor quality. The commonly associated keywords are flexibility, stretchability, waterproofness, transparency, and self-healing. Various nanomaterials and designs have been used to increase sensor sensitivity. Sensors are commonly fabricated from silver and gold nanoparticles, graphene, and CBTs. Liu et al. placed a woven fabric in a graphene sensor and achieved a gauge factor (GF) as high as 223 [94]. Yan et al. electrospun a nanofiber yarn made of carbon black and graphene nanocomposites [95], achieving a GF of 1700. Chen et al. reported a GF of 4000 using a silver nanowire and graphene [96]; the sensor exploited mechanical cracking. Sensors are now additionally 
flexible, stretchable, and (sometimes) waterproof (Table 1). The most popular appended properties are flexibility and stretchability, and the sensors can be placed on free-formed or deformable surfaces. Flexibility and stretchability also increase the sensing range. Flexibility is achieved by the use of nanoparticles [61, 62, 64, 65] or graphene [63]. Su et al. printed silver nanoparticles and fabricated a liquid channel (to introduce flexibility), achieving a GF of 3000 [64]. Stretchability was achieved by printing nanoparticles [74] or graphene [70] onto stretchable substrates. In many cases, nanomaterials are mixed or specifically designed to maximize GF and extend stretchability. Zhang et al. used nanocomposites of silver nanoparticles and CNTs to attain a stretchability of $95 \%$ and a GF of 39.8 [66]. Jeong et al. developed fragmented, structured strain sensors made from graphene foam with a stretchability of 70\% [73]. Guo et al. created a sandwich strain sensor fabricated from CNTs and a carbon black nanocomposite, achieving a stretchability of $120 \%$ [72]. Park et al. used a graphene yarn to develop a sensor with a stretchability of $150 \%$ and a sensitivity of 10 [67]. Zhou et al. increased the sensitivity of a $50 \%$ stretchable sensor fabricated using CNTs to 100,000 , exploiting mechanical cracking [68]. Zhao et al. extended the stretchability to $120 \%$, with a GF of $10,000,000$, by combining graphene and silver nanoparticles in a fragmented structure [75].

The sensor performance typically varies with pressure, temperature, and humidity. Temperature, in particular, exerts major effects; nanomaterials are temperature-sensitive because of their size (Fig. 2). The sensor performance must be independent of the temperature unless the sensor is to measure the temperature. Yi et al. fabricated a temperaturetunable sensor using a thermally evaporated gold nanofilm [82]. Rahman et al. created a three-dimensional (3D), printed, silver, nanoparticle sensor stable at $500{ }^{\circ} \mathrm{C}$ [81].
Table 1 Research trends in sensing applications

\begin{tabular}{|c|c|c|c|c|}
\hline $\begin{array}{l}\text { Original } \\
\text { physics }\end{array}$ & $\begin{array}{l}\text { Original } \\
\text { property }\end{array}$ & $\begin{array}{l}\text { Appended } \\
\text { property }\end{array}$ & $\begin{array}{c}\text { Appended } \\
\text { physics }\end{array}$ & Ref. \\
\hline \multirow{10}{*}{$\begin{array}{l}\text { Strain } \\
\text { sensing }\end{array}$} & \multicolumn{3}{|l|}{ Sensitivity } & \\
\hline & & \multicolumn{2}{|l|}{ Flexibility } & [61-65] \\
\hline & & \multicolumn{2}{|l|}{ Stretchability } & [66-75] \\
\hline & & \multicolumn{2}{|l|}{ Waterproof } & [76-78] \\
\hline & & \multicolumn{2}{|l|}{ Self-healing } & \\
\hline & & \multicolumn{2}{|l|}{ Transparency } & {$[69,79,80]$} \\
\hline & & \multicolumn{2}{|c|}{ Temperature independence } & {$[81,82]$} \\
\hline & & & $\begin{array}{l}\text { Wireless } \\
\text { communication }\end{array}$ & {$[83,84]$} \\
\hline & & & Structural colour & [85-88] \\
\hline & & & Multi-sensing & [89-92] \\
\hline \multirow{4}{*}{$\begin{array}{l}\text { Pressure } \\
\text { sensing }\end{array}$} & \multicolumn{3}{|l|}{ Sensitivity } & \\
\hline & & \multicolumn{2}{|l|}{ Flexibility } & \\
\hline & & \multicolumn{2}{|l|}{ Transparency } & [93] \\
\hline & & & Multi-sensing & [74] \\
\hline Temperatur & \multicolumn{3}{|l|}{ Sensitivity } & \\
\hline e sensing & & \multicolumn{2}{|l|}{ Flexibility } & \\
\hline
\end{tabular}




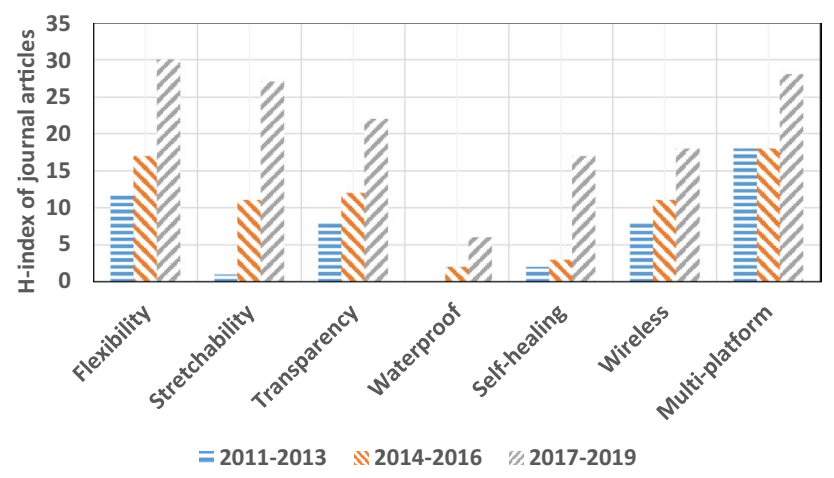

Fig. 10 H-index trajectory of keywords relevant to high-sensitivity sensors and their appended properties used between 2011 and 2013, 2014-2016, and 2017-2019. Data were obtained from the Web of Science in December 2019

Furthermore, sensors have been often rendered transparent. Lee et al. developed a sensor with $90 \%$ transmittance. Silver nanoparticles assume the shape of a sea urchin during spincoating [93] and waterproofing was required prior to practical use. Jeon et al. created a waterproof sensor using silver nanoparticles and CNTs [76]. Sensors fabricated from sputtered gold films featuring mechanical cracks [78], and from carbon black and yarn [77], have been waterproofed. Finally, in the last step of multi-functionalization, some sensors have explored new physics. Roh et al. studied a sandwich strain sensor (fabricated from CNTs) that was both stretchable and transparent. The GF was 62 , and the stretchability and transmittance were $100 \%$ and $62 \%$, respectively [69]. Shi et al. used CNTs and a graphene network to develop transparent stretchable sensors [81]. Liao et al. fabricated a multifunctional sensor from a zinc oxide $(\mathrm{ZnO})$ nanowire; the sensor measured temperature, ultraviolet light level, and strain. The GF was 15.2 [92]. Furthermore, wireless communication is also possible; electromagnetic methods use radiofrequency (RF) signals $[83,84]$ or structural color [85-88], as will be discussed in Sect. 4.3.

The self-healing property is the highest increasing property in terms of sensor research trends using nanomaterials. Self-healing materials take inspiration from biological systems that can self-organize and self-repair. Self-healing materials would reduce the need for costly maintenance by healing themselves when damage occurs. One example includes ingredients that are automatically released or expand to fill cracks when they appear. Because of the increasing cost of the sensors and their properties, which are difficult to maintain in case of failure due to miniaturization, it is expected that research on self-healing properties will continue.

\subsection{Actuators}

An actuator converts stimuli such as light, heat, and electrical and magnetic fields into a mechanical output. Electrostatic, piezoelectric, shape memory alloy (SMA), thermal, electrochemical [107, 108], and photo actuators have been described. We focus on electrical actuators, where an electric signal triggers a mechanical output. Such actuators have various applications (for example, artificial muscles). The outputs differ in terms of displacement, force, and frequency. Most actuators are flexible, allowing deformation or deflection; however, we do not consider flexibility to be an appended property. Nanomaterials including nanoparticles [109-111], carbon black [112], CNTs [113, 114], and graphene [115-119] have been used to fabricate actuators. Lu et al. used CNTs to develop a micro-optomechanical actuator via photolithography, achieving a maximum deflection of $22 \mathrm{~mm}$ [114]. Sul et al. fabricated a bimorphic actuator using multi-walled CNTs (MWCNTs) and aluminum [120]. Liang et al. prepared an electromechanical actuator by stacking graphene sheets fabricated via filtration. $\mathrm{Fe}_{3} \mathrm{O}_{4}$ nanoparticles were placed between the sheets to enhance their performance [117]. Park et al. developed an actuator triggered by humidity that featured a bilayer of graphene oxide and MWCNTs [118]. Li et al. created an actuator exhibiting $360^{\circ}$ deflection using a reduced graphene oxide (rGO)/polydimethylsiloxane (PDMS) bilayer and direct ink printing [119]. Kong et al. developed an ionic polymer metal composite (IPMC) actuator employing CNTs and graphene [121].

The properties of the actuators are listed in Table 2, and Fig. 11 shows the research trends revealed by the H-index of journal keywords. The most popular keyword in terms of an appended property is "soft". Polymers are mixed with nanomaterials. The actuation mechanism differs based on the material used. Photoactuators (activated by light) were created by mixing photoresponsive nanoparticles with poly(N-isopropylacrylamide) (PNIPAAm) (a thermally sensitive hydrogel) [97, 98, 103, 122]. Sun et al. developed a very responsive photoactuator (deflection range of $180 \%$ ) using titanate nanosheets, gold nanoparticles, and PNIPAAm [103]. Deng et al. fabricated an anisotropic photomechanical actuator that enabled multidirectional actuation. The bilayer structure featured a CNT/paraffin wax composite and polyimide [122]. Many thermal actuators have been prepared by mixing nanomaterials with PNIPAAm [100, 102]. Kim et al. mixed a titanate nanosheet with PNIPAAm to develop a thermal actuator with a deflection range of $170 \%$ and a response time of $1 \mathrm{~s}$ [102]. Furthermore, nanomaterials have been used to fabricate electrical actuators. Yang et al. used rGO and poly(2-acrylamido-2-methylpropanesulfonic acid) (PAMPS) to develop an electrical actuator [101]. Some actuators are triggered by humidity. Gladman et al. created such an actuator using nanofibrillated cellulose and 
$\mathrm{N}$, N-dimethylacrylamide, employing various geometries (swollen, twisted, spiral, or helicoidal) to study the actuation characteristics [99]. The other property attributed to actuators is transparency (Table 2). Zhu et al. used graphene and epoxy to create a bimorphic structure via chemical vapor deposition, photolithography, and reactive ion etching. The transparent actuator mimics the shape and motion of the fly wing. The maximum deflection range was $4.5 \mu \mathrm{m}$ [104].

Multi-functionalization has also been realized by rendering actuators multi-responsive. Han et al. developed an actuator that reacts to humidity, light, and electrical currents. MWCNTs, carbon nanofibers, and poly(3,4-ethylenedioxythiophene) polystyrene sulfonate (PEDOTPSS) were printed via electrospinning [105]. Shepherd et al. placed molecular spin-crossover materials in a polymer matrix when preparing a multi-responsive actuator in response to both temperature and light [106].

Among the important properties of actuators, force and frequency are greatly affected by the weight of the actuator itself, and therefore, efforts to reduce the weight itself are expected to continue. Manufacturing composite materials composed of nanomaterials is a widely known technique for achieving lightweight materials. In addition, considering the industry to which actuators are applied, advanced composites can help build strong, lighter components for vehicles, including aircrafts. In addition to next-generation nanocomposites, ongoing advances in composites made from carbon fiber and other materials could make it possible to substitute composites for materials such as aluminum in increasingly more applications. These advances include new methods

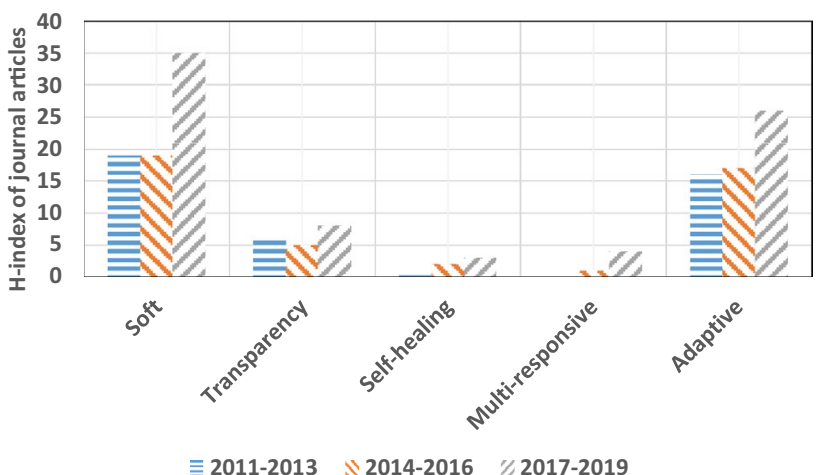

Fig. $11 \mathrm{H}$-index trajectory of keywords related to high-force actuators and their appended properties used between 2011 and 2013, 20142016, and 2017-2019. Data were obtained from the Web of Science in December 2019

for producing and binding carbon fibers, allowing for less expensive fabrication.

In addition, memory metals that revert to a prior shape when heated to a specific temperature could be a direct method for self-adaptation to the surrounding environment. These materials are being considered as a way of producing movement in an actuator using a charge to expand or contract the materials, imitating muscle movement. Some versions of memory metals can be programmed to take on multiple shapes at different temperatures.
Table 2 Research trends in actuator applications

\begin{tabular}{|c|c|c|c|c|}
\hline $\begin{array}{l}\text { Original } \\
\text { physics }\end{array}$ & Original property & $\begin{array}{l}\text { Appended } \\
\text { property }\end{array}$ & $\begin{array}{l}\text { Appended } \\
\text { physics }\end{array}$ & Ref. \\
\hline \multirow[t]{7}{*}{ Actuation } & \multicolumn{3}{|c|}{ Force/Displacement/Frequency } & \\
\hline & & \multicolumn{2}{|l|}{ Soft } & [97-103] \\
\hline & & \multicolumn{2}{|c|}{ Transparency } & [104] \\
\hline & & \multicolumn{2}{|l|}{ Waterproof } & \\
\hline & & \multicolumn{2}{|l|}{ Self-healing } & \\
\hline & & & $\begin{array}{l}\text { Multi } \\
\text { responsive }\end{array}$ & {$[105,106]$} \\
\hline & & & $\begin{array}{l}\text { Adaptive } \\
\text { system }\end{array}$ & \\
\hline
\end{tabular}




\subsection{Energy Harvesters}

Nanomaterials may reduce our reliance on traditional energy sources and help meet increasing energy demands by allowing us to use clean, affordable, and renewable energy sources and reduce energy consumption. Thin-film solar panels attached to computer cases and flexible piezoelectric nanowires woven into clothing generate usable energy from light, friction, and body heat, thus powering mobile electronic devices. Similarly, various nanoscience-based approaches are aimed at converting the waste heat of computers, automobiles, homes, and power plants into usable electric power. Energy-efficient and energy-saving products are increasing in both number and type. Nanotechnology may yield affordable, clean drinking water via rapid low-cost detection and treatment of impurities.

The trend shows that efforts are underway to append new properties to energy-harvesting devices. Table 3 and Fig. 12 list and show the trends in properties attributed to piezoelectric and triboelectric energy-harvesting systems. Flexibility is often imparted for piezoelectric applications. A piezoelectric device converts mechanical energy into electric energy, and vice versa. Flexibility thus expands the device utility. Many researchers have developed flexible piezoelectric energy harvesters using nanomaterials such as $\mathrm{ZnO}$, lead titanate $\left(\mathrm{PbTiO}_{3}\right)$, lead zirconate titanate (PZT), and polyvinylidene fluoride (PVDF) as nanoparticles [128, 130, 132, 138], nanofibers [125, 129, 131], nanotubes [123], or nanosheets [124, 126]. Liu et al. developed a flexible piezoelectric energy harvester by electrospinning PVDF nanofibers and lead oxide nanoparticles [125]. Shin et al. screen-printed barium zirconium titanate (BZT) nanoparticles onto polyvinylidene fluoride (PVDF) nanofilms to ensure flexibility. The energy density was $2.79 \mathrm{~mJ} / \mathrm{cm}^{3}$ [126]. Shi et al. created a flexible piezoelectric energy harvester with an open-circuit voltage of $11 \mathrm{~V}$ from graphene nanosheets and barium titanate $\left(\mathrm{BaTiO}_{3}\right)$ nanoparticles [124]. Flexible harvesters have also been electrospun from nanocomposites of titanate nanoparticles/PVDF nanofibers [129] and nickel oxide (NiO) nanoparticles/silicon oxide $\left(\mathrm{SiO}_{2}\right)$ nanoparticles [130]. Jeong et al. reported a stretchability of $200 \%$ using lead magnesium niobate-lead titanate (PMN-PT) nanoparticles, silver nanowires, and CNTs [123]. Nanomaterials have also been used to generate triboelectric energy generators $[138,140]$. Chen et al. employed strontium titanate $\left(\mathrm{SrTiO}_{3}\right)$ nanoparticles with nanopores to fabricate a flexible triboelectric energy device. The opencircuit voltage and power density were $338 \mathrm{~V}$ and $6.47 \mathrm{~W} /$ $\mathrm{m}^{2}$, respectively [138].

As the third step of multi-functionalization, new physics was introduced. A multifunctional energy harvester exploiting both piezoelectric and triboelectric effects was developed using PVDF nanoparticles dispersed in a leaf-shaped structure [139]. $\mathrm{PbTiO}_{3}$ and PZT are commonly used for piezoelectric energy harvesting. However, these materials contain lead, which is toxic. Therefore, lead-free energy harvesters have attracted considerable attention. Baek et al. used barium titanate nanoparticles and a nanowire composite; a
Table 3 Research trends in energy harvesting applications

\begin{tabular}{|c|c|c|c|c|}
\hline $\begin{array}{c}\text { Original } \\
\text { physics }\end{array}$ & $\begin{array}{l}\text { Original } \\
\text { property }\end{array}$ & $\begin{array}{l}\text { Appended } \\
\text { property }\end{array}$ & $\begin{array}{c}\text { Appended } \\
\text { physics }\end{array}$ & Ref. \\
\hline \multirow[t]{6}{*}{ Piezoelectric } & \multicolumn{3}{|c|}{ Output voltage/current } & \\
\hline & & \multicolumn{2}{|l|}{ Stretchability } & {$[123]$} \\
\hline & & \multicolumn{2}{|l|}{ Flexibility } & [124-132] \\
\hline & & & Transparency & {$[133]$} \\
\hline & & & Self-clean & {$[134]$} \\
\hline & & & Lead-free & [135-137] \\
\hline \multirow[t]{3}{*}{ Triboelectric } & \multicolumn{3}{|c|}{ Output voltage/current } & \\
\hline & & \multicolumn{2}{|l|}{ Flexibility } & {$[138]$} \\
\hline & & & $\begin{array}{l}\text { Multiphysics } \\
\text { (Piezoelectric) }\end{array}$ & [139] \\
\hline
\end{tabular}




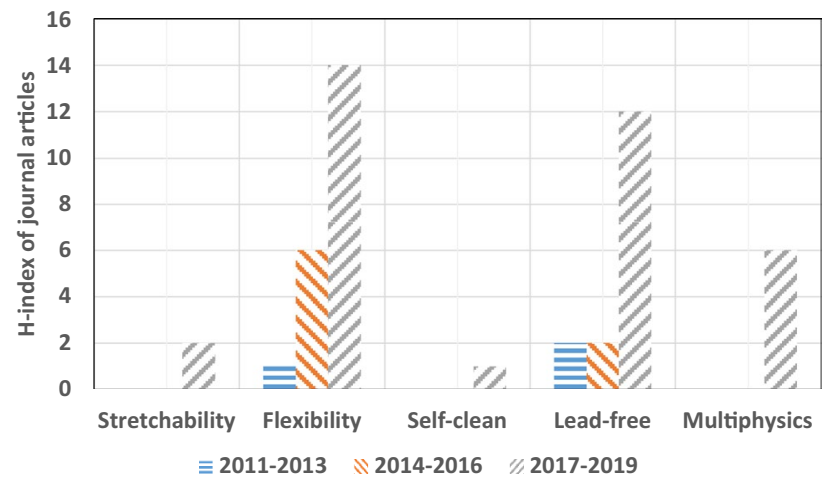

Fig. 12 H-index trajectory of keywords related to high-sensitivity energy harvesters and their appended properties used between 2011 and 2013, 2014-2016, and 2017-2019. Data were obtained from the Web of Science in December 2019

hydrothermal process was employed to create a flexible, lead-free energy harvester [135]. Gupta et al. created a lead-free, flexible piezoelectric device using microcubes of sodium potassium lithium niobate (NKLN) [136]. Kar et al. appended a self-cleaning property to a flexible piezoelectric device using a tin oxide $\left(\mathrm{SnO}_{2}\right)$ nanosheet [134]. Wang et al. developed a transparent energy harvester with a peak power density of $17.73 \mathrm{~W} / \mathrm{m}^{2}$ using gold nanoparticles [133].

One of the main objectives of energy harvesters is to harness energy from its surrounding environment and store this harnessed energy for later use. We expected that research on energy harvesters would be more than contributing to the environment by simply saving and generating energy. Because piezoelectric materials that turn pressure into electricity are not new and have reached technical limitations in terms of efficiency, efforts to change the materials and structures themselves into eco-friendly materials are likely to be attempted. Green materials that attempt to solve environmental issues in a more direct manner are estimated to account for low carbon-dioxide emissions. Furthermore, the lead-free property could be an existing example of researchers' efforts toward going green.

\subsection{Solar Cell}

Energy storage technology includes batteries and other systems that store energy for later use. Lithium-ion (Li-ion) batteries and fuel cells are already powering electric and hybrid vehicles and billions of portable consumer electronic devices. Li-ion batteries, in particular, have improved greatly in terms of performance and fall in price; the cost per unit of storage capacity has declined considerably over the past decade. Over the next 10 years, advances in energy storage technology may render electric vehicles (hybrids, plug-in hybrids, and all-electronic vehicles) cost-competitive with vehicles using internal combustion energy. In power grids, advanced battery storage systems integrate solar and wind power, improve power quality by controlling frequency variations and handling peak loads, and reduce costs by enabling utilities to postpone infrastructure expansion. In developing economies, battery/solar systems may deliver reliable power to places that have never had power. Here, we focus on solar cells as an example of multi-functionalization. The original property is energy density. The materials used for construction include silver [144], titanate [145-147], gold [148], and $\mathrm{ZnO}$ nanoparticles [149], among others [150-153]. A photovoltaic device with a magnesium oxide nanolayer afforded efficient (18.23\%) power conversion [154].

Solar cells have become flexible [141] and transparent [142] (Table 4). Figure 13 shows the related research trends. $\mathrm{Zi}$ et al. developed a flexible photovoltaic device with a photocurrent density of $3.0 \mathrm{~mA} / \mathrm{cm}^{2}$ using silver nanoparticles in a hydrogenated, amorphous silicon/germanium base [141]. Bush et al. fabricated semi-transparent photovoltaic devices using aluminum and zinc oxide nanoparticles [142]. Solar cells exhibiting multi-physics have been studied from the viewpoint of multi-functionalization. Dou et al. used bismuth tellurium nanotube/ $\mathrm{ZnO}$ nanoparticle composites to convert solar energy into both thermal and photo energy, thus enhancing the solar cell efficiency [143].

Recently, metamaterials that revert their original properties, including permittivity, permeability, emissivity, and thermal/ electrical conductivity by light, have been introduced. They have the advantage that they can be applied to applications that include a solar cell in a compact manner because it may not require additional equipment or physical sources that are required to change various intrinsic properties.

\section{Case Study: Sensing Application}

Sensing applications, usually termed sensors, are actively researched by those interested in advanced nanomaterials. Since 1950, efforts have been made to explain the physical behaviors of nanomaterials used in sensing applications. Usually, simplified models based on quantum mechanics, or molecular dynamic or simulation methods, have been employed to model crowded nanomaterial environments. Recently, such approaches have provided valuable guidance to those interested in the synthesis and exploitation of novel nanomaterials. State-of-the-art sensors use nanoparticles, nanotubes, and graphene to exploit the physical phenomena associated with the scale effects of nanomaterials (absent from bulk materials), to increase sensor performance, and to expand their potential applications. The scale of the nanomaterials can be found in Fig. 1 in the introduction.

In the present era of big data, sensors are finding applications that were unimaginable earlier. Wearable sensors measure human body motion, control human-machine 
Table 4 Research trends in solar cell applications

\begin{tabular}{|c|c|c|c|c|}
\hline $\begin{array}{l}\text { Original } \\
\text { physics }\end{array}$ & $\begin{array}{l}\text { Original } \\
\text { property }\end{array}$ & $\begin{array}{l}\text { Appended } \\
\text { property }\end{array}$ & $\begin{array}{c}\text { Appended } \\
\text { physics }\end{array}$ & Ref. \\
\hline \multirow{5}{*}{ Photovoltaic } & \multicolumn{3}{|l|}{ Energy density } & \\
\hline & & \multicolumn{2}{|l|}{ Flexibility } & [141] \\
\hline & & \multicolumn{2}{|l|}{ Stretchability } & \\
\hline & & \multicolumn{2}{|l|}{ Transparency } & [142] \\
\hline & & & $\begin{array}{l}\text { Multiphysics } \\
\text { (thermoelectric) }\end{array}$ & [143] \\
\hline
\end{tabular}

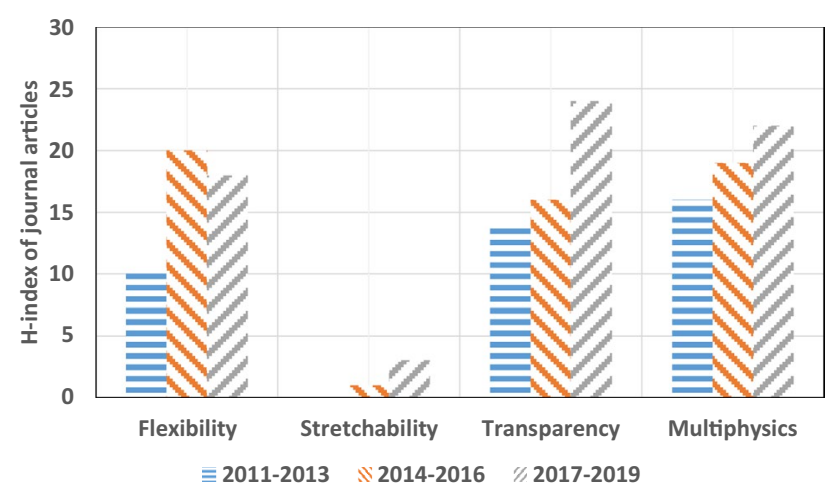

Fig. 13 H-index trajectory of keywords related to high-energy density solar cells with additional properties used between 2011 and 2013, 2014-2016, and 2017-2019. Data were obtained from the Web of Science in December 2019

interfaces during manufacturing, and monitor manufacturing robots. Sensors must be increasingly flexible, stretchable, and communicative (Fig. 14), and it is expected that future applications may be even more demanding. Here, we deal with research trends through the lens of our multi-functionalizing strategy. Sensor performance indicators include sensitivity, stretchability, measurement range, mechanical durability, signal linearity, and hysteresis; however, here we focus on the first three, as these are the most relevant. Furthermore, we explore only one type of sensor that finds applications in biology, medicine, mechanical engineering, and manufacturing.

A strain sensor detects the strain caused by stretching, bending, or twisting and emits an electrical signal (Fig. 15). Such sensors may be resistive, capacitive, or piezoelectric (Fig. 16) [155]. The piezoresistive sensor detects a change in resistance and is fabricated from conductive materials such as graphene, CNTs, metal nanoparticles, or a conductive polymer. The resistance changes on mechanical deformation and the piezocapacitive sensor detect the associated capacitance change. The basic sensor features conductors and a dielectric. When strain develops, the distance between the conductors (and hence the capacitance) changes. The piezoelectric sensor detects the electrical charges produced. Piezoelectric materials generate charges on mechanical deformation, and either the voltage or current can be measured. Sensitivity is usually defined by reference to how external stimuli change sensor properties. The sensitivity of a piezoresistive sensor is the extent of the external force required to induce a measurable resistance change. GF is often used to compare such sensors, and is

$\mathrm{GF}=\frac{\Delta R / R}{\varepsilon}$

where $\Delta R$ is the increase in the sensor resistance compared to the initial resistance $R$ and $\varepsilon$ of the induced strain. As it is difficult to compare the performances of sensors that differ in terms of sensing mechanisms, we focus only on the piezoresistive sensors below.

Figure 17 and Table 5 show the multi-functionalization research trends among studies that studied piezoresistive strain sensors. Below, the methods used to increase the sensitivity and add stretchability are discussed in detail. Wireless communication by sensors is discussed in Sect. 4.3.

\subsection{Increasing Performance: Toward High Sensitivity}

Sensors exploit geometrical changes, such as cracking, percolation, and tunneling. Figure 18 shows the sensing mechanisms of the $0 \mathrm{D}, 1 \mathrm{D}$, and $2 \mathrm{D}$ nanomaterials. The latter two materials exhibit more stable electrical connections under strain. As both graphene and CNTs exhibit excellent electrical conductivity and stability, they are frequently used in sensor applications requiring high sensitivity $[174,180,187$, 188]. The scale of these materials is in tens of nanometers in 


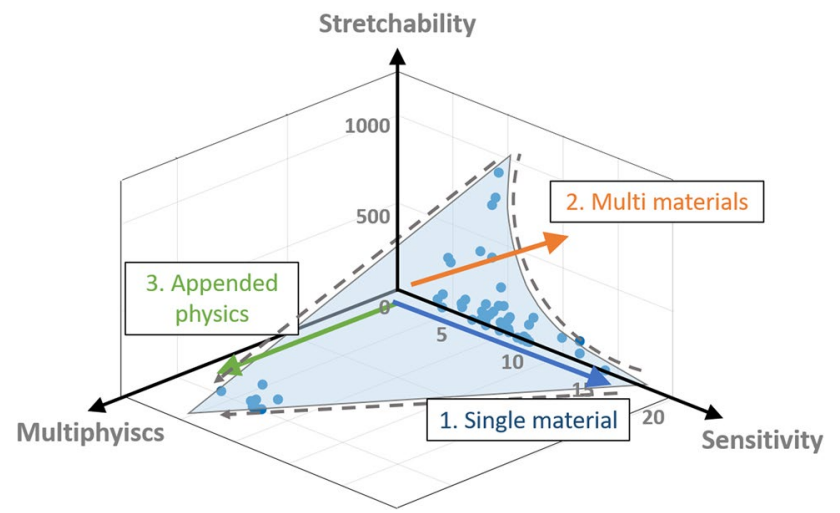

Fig. 14 Multi-functionalization of nanomaterials used for sensing applications: increasing sensitivity, adding stretchability, and achieving wireless communication

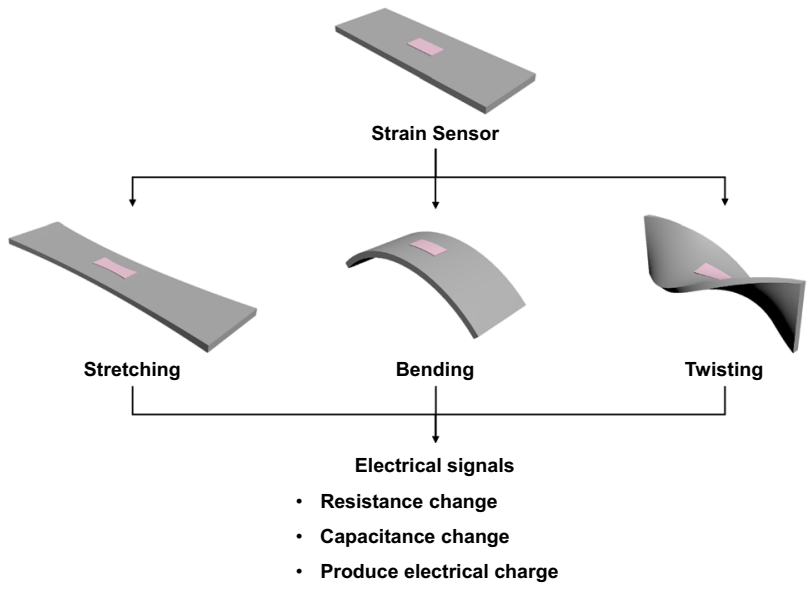

Fig. 15 A schematic showing how mechanical strain is converted into an electric signal after various mechanical deformations

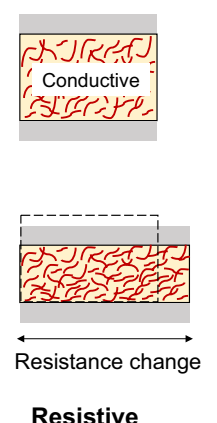

Resistive

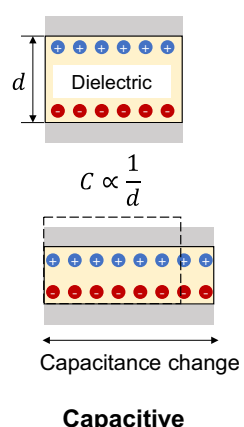

Capacitive
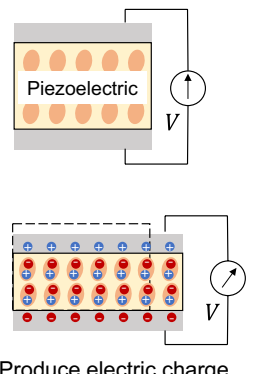

Produce electric charge

Piezoelectric
Fig. 16 A schematic showing the sensing mechanisms of resistive, capacitive, and piezoelectric sensors (modified from [155])

diameter and a few to tens of micrometers in length. To further increase the sensitivity, graphene and CNTs are mixed with other nanomaterials $[172,181,182,184]$ or combined to form auxetic [177] or yarn [67] structures. Rahim et al. mixed graphene and CNTs and achieved a GF of 20,000 [182]. Xiao et al. reported a higher GF $(1,000,000)$ using a nanocomposite of graphene and copper mesh [181].

Zero-dimensional materials exhibit fewer and shorter electrical connections when stretched compared with 1D and 2D materials. However, metal nanoparticles that have a diameter of a few to hundreds of nanometers have recently been used to increase sensor GFs. For example, Zhao et al. fabricated a sensor from silver nanoparticles and graphene; the GF achieved was 100,000 [75]. Metal nanoparticles are added not because of their intrinsic electrical properties, but rather because of changes in the nanogaps between the nanoparticles. In contrast to a typical commercial strain gauge featuring a bulk metal thin film, the resistance changes with an increase in length (because of the associated thinning of the crosssectional area) governed by Ohm's law, the contact resistance between nanoparticles increases dramatically as the nanogap increases. An electron tunneling model is used to guide the development of nanoparticle synthesis (in terms of diameter and homogeneity); due to this, sensitivity increases further. However, because the connection is unstable, stretchability is limited when only OD materials are employed. There is a trade-off between sensitivity and stretchability, which is discussed in Sect. 4.2. Sensors exploiting mechanical cracking are similar to those of nanoparticle sensors. When strain is applied, a mechanical crack creates a nanogap, greatly changing the resistance and thus imparting high sensitivity. Zhou et al. exploited mechanical cracking when fabricating sensors from CNTs [68].

In electromechanical sensing applications, which is the scope of this section, the main advantage of using nanomaterials is their higher electrical conductivity and sensitivity. In nanomaterials, the electron mean free path is in a similar range as the diameter; therefore, the quantum confinement effect occurs. The electrical properties differ from those of bulk materials. Therefore, nanomaterials enable faster and higher performance. Both graphene and CNTs have excellent electrical conductivity. As discussed, sensors fabricated with OD materials can have high sensitivity with nanogaps and electron tunneling effects. Graphene and CNTs also consist of structures with high porosities, which can increase the sensitivity. The materials themselves have a high piezoresistive gauge factor.

In addition, the performance of sensors can be easily designed through appropriate material selection. For nanoparticles, as the diameter decreases, the electromagnetic properties change accordingly. The bandgap of nanowires varies with their wire diameter, and their electrical properties can be tuned by doping. The properties of CNTs depend 
Fig. 17 Research trends of piezoresistive strain sensors with respect to materials

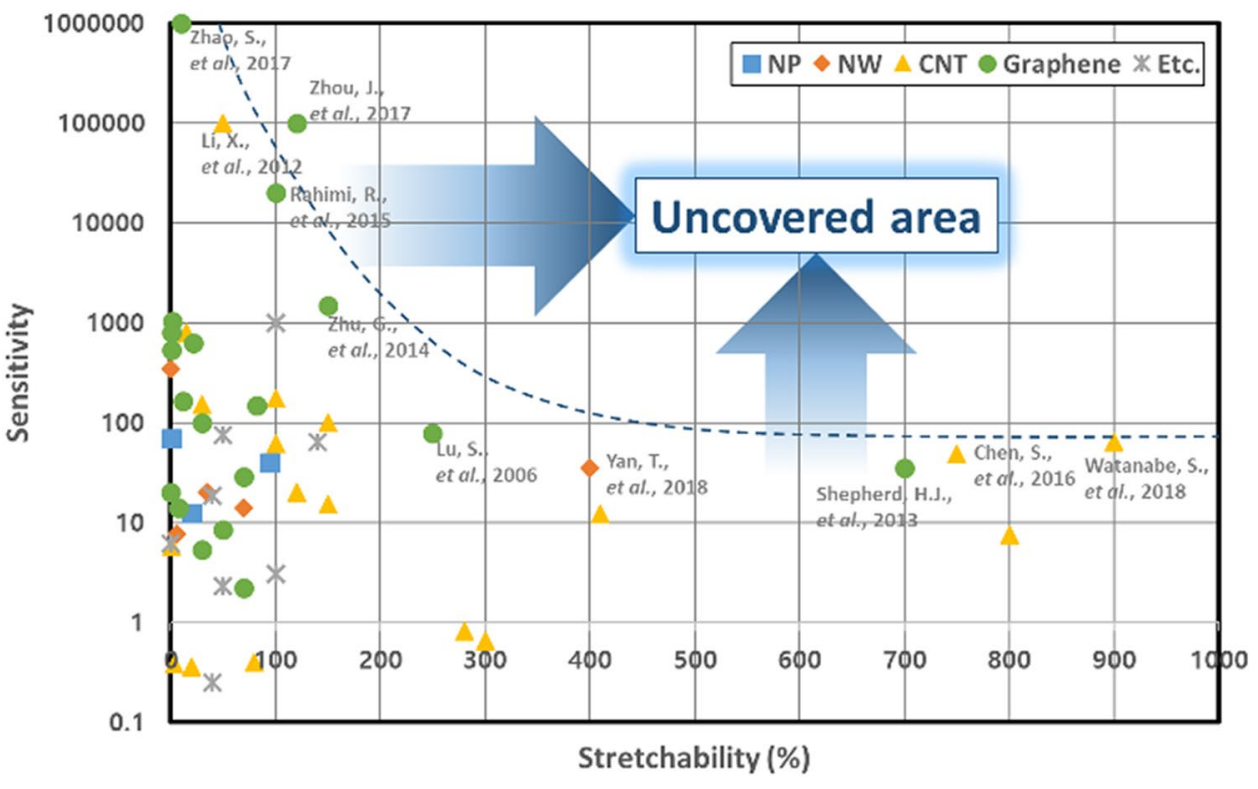

on their diameter, length, crystallinity, surface, number of walls, and shape.

Furthermore, nanomaterials have stronger mechanical properties than bulk materials. Therefore, sensors fabricated with nanomaterials can exhibit higher mechanical properties with light weight. The good adhesion of nanomaterials can be another advantage of using nanomaterials. When printing sensors using the bottom-up method, the patterns should attach firmly to the substrate to increase stability. As nanomaterials have a high surface energy and maintain good adhesion with the substrate, long-term stability can be achieved.

Another advantage of nanomaterials is that they have a high reaction rate because of their high surface-to-volume ratio. A large number of broken bonds increase the surface reactivity. They normally have a lower activation energy than the bulk materials. Chemical and bio-sensing applications utilize these characteristics to increase the number and speed of reactions in sensors. The number of effective reaction areas increases, resulting in higher sensitivity.

\subsection{Appending Properties: OVERCOME the Trade-Off Between Sensitivity and Stretchability}

Given the increasing demand for wearable, skin-mountable devices that measure human body motion, respiration, and voice, current strain sensors must be flexible and stretchable. Stretchability increases the measuring range and is important if the deformations are large. In addition, soft actuators and soft robotics require stretchable sensors and electronics.

Three strategies have been used to enhance stretchability. The first employs 1D or 2D nanomaterials that are very stretchable because their longitudinal components are (obviously) greater than those of 0D materials; they remain conductive under strain (Fig. 18). Zhang et al. used a carbonized, plain weave cotton fabric on an Ecoflex substrate to increase the stretchability to $140 \%$ [164]. Liao et al. increased the stretchability to $150 \%$ using CNTs and polyurethane (PU), employing a soak coating/hydrothermal method to create textiles [92]. Liu et al. described a sensor with GF and stretchability of $100 \%$ and $150 \%$, respectively. Single-walled CNTs (SWCNTs) were allowed to self-assemble on a PDMS substrate [175]. Yamada et al., using a similar approach, achieved a stretchability of $280 \%$ [166]. Two-dimensional materials are even more stretchable. Cheng et al. used graphene to develop a strain sensor with a stretchability of $700 \%$ [178]. Furthermore, liquids can be used to fabricate stretchable strain sensors. Chossat et al. fabricated a $100 \%$ stretchable strain sensor employing an ionic solution and eutectic gallium-indium (EGaIn) Ecoflex substrate [190].

The second method employs nanocomposites. Metal nanoparticles may crack when used to measure large deformations. Strain measurement is very sensitive, but only within a limited range [156, 157]. Hence, CNTs and graphene have been used to create sensors with high aspect ratios that are mechanically stiff and electrically conductive (Fig. 19). CNTs and graphene exhibit features that render them especially suitable for stretchable sensing, but are not very sensitive. Hence, nanocomposites of both metal nanoparticles and CNTs or graphene have been formulated to ensure both high sensitivity and stretchability. Zhang et al. increased the stretchability by up to $95 \%$ by mixing silver nanoparticles with CNTs [66]; stretchability was greater than that imparted by nanoparticles alone. Zhao et al. combined AgNPs with 


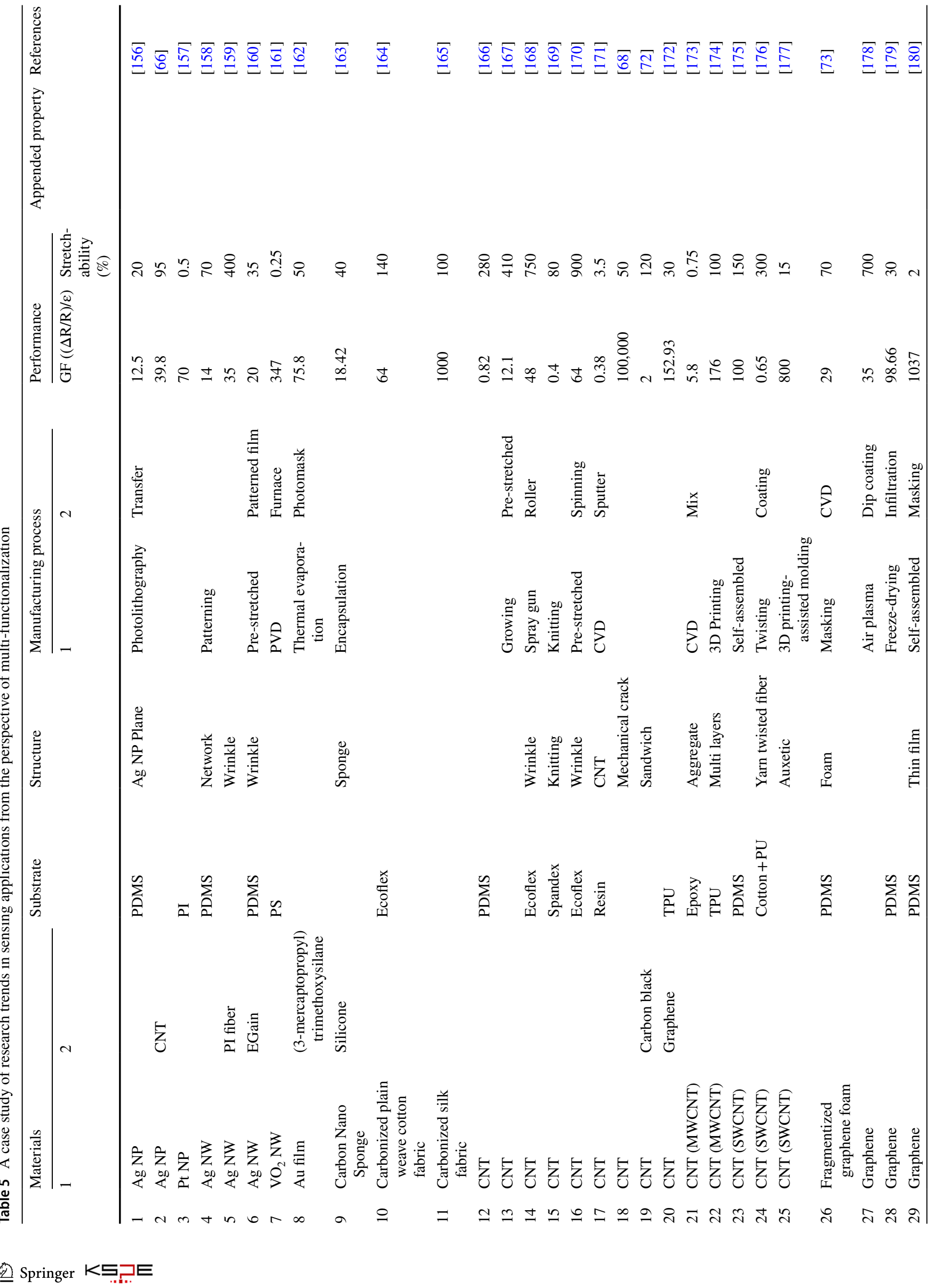




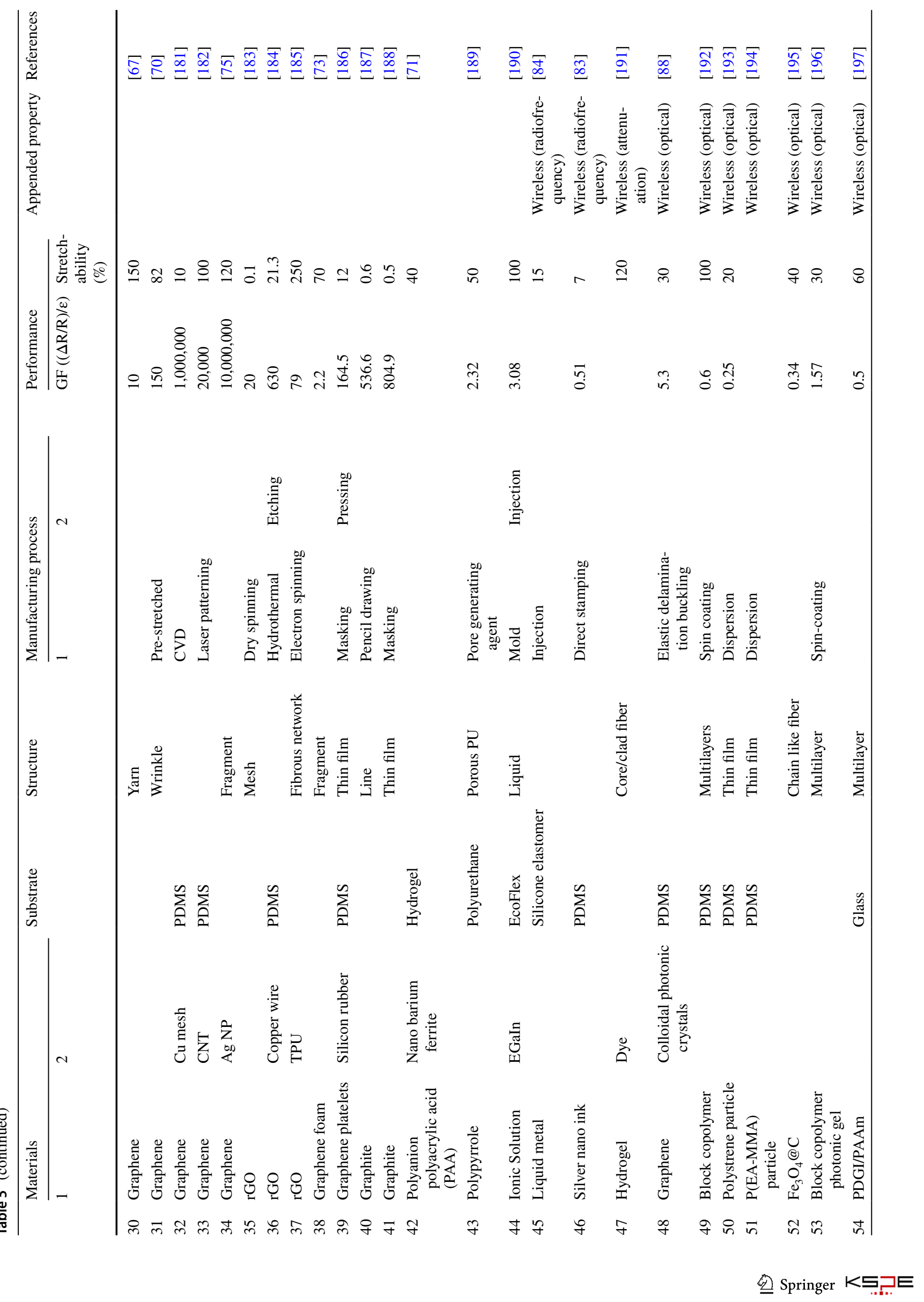




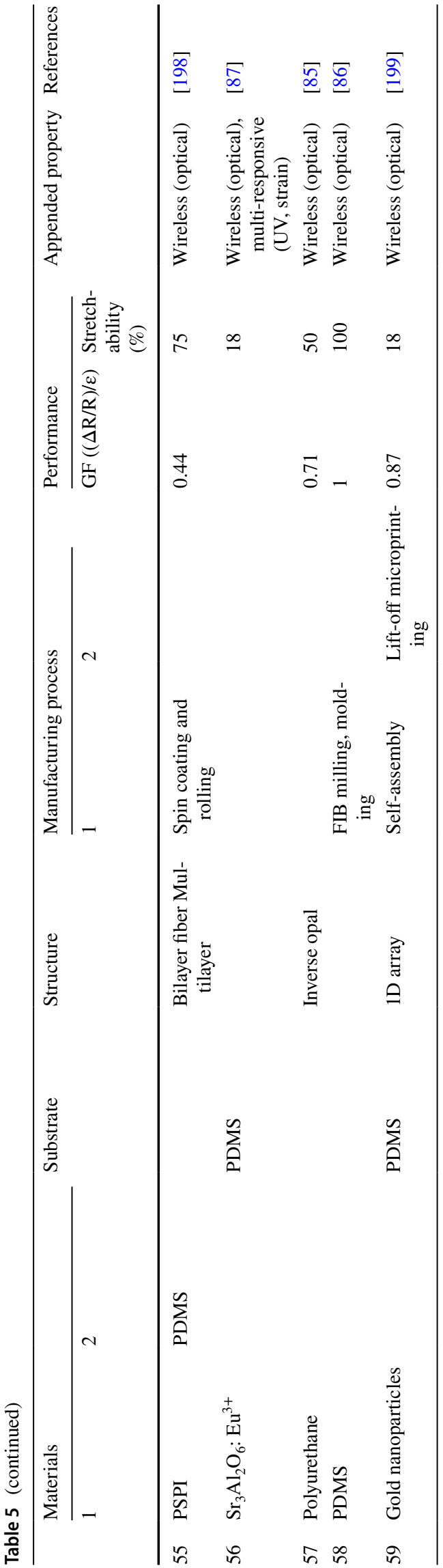

graphene to ensure high sensitivity and stretchability. A fragment structure was employed to maximize sensitivity. The GF was 100,000, and the stretchability was $120 \%$ [75]. Guo et al. mixed CNTs with carbon black, and the sensor stretchability was $120 \%$ [72]. The nanoparticles imparted sensitivity and the CNTs and graphene stretchability; the nanocomposite exploits both features via a mechanical or chemical reaction. However, a trade-off is in play; sensitivity or stretchability predominates depending on the composition ratio of the two nanomaterials. Advanced manufacturing processes were used to reduce this trade-off.

Finally, designs that endure many applications of strain increase stretchability. The most popular structure is wrinkles [70, 159, 167, 168, 170]. Figure 20a shows the fabrication process. A stretchable substrate is pre-stretched during material deposition and, on release, forms wrinkles that can be initially stretched flat and then further stretched. Wei et al. fabricated a wrinkled structure from a silver nanowire and polyimide fiber with a stretchability of $400 \%$ [159]. Park et al. reported a higher stretchability (750\%) using CNTs on an Ecoflex substrate to create wrinkles [168]. Ryu et al. used a similar approach and achieved a stretchability of $900 \%$ [170]. Yarns and fiber networks (especially the latter) have been widely studied $[67,176]$. Figure $20 \mathrm{~b}$ shows the shape of the sample yarn. This is durable under strain because the fibers are strongly interwoven. Foroughi et al. twisted SWCNTs and PU into yarn with a stretchability of 300\% [176]. Wang et al. electrospun a fibrous network using rGO and thermoplastic polyurethane. The stretchability was $250 \%$, and the GF was 79 [185]. Wang et al. woven MWCNTs and rubber fibers into textiles; the stretchability was $800 \%$ [89].

Likewise, three strategies exist for achieving stretchability; here we analyze each strategy. The use of nanomaterials with length components is the most popular and effective method. Graphene and CNTs are the most frequently used materials based on a literature review. This is because they not only have high electrical conductivity and strong mechanical properties, but also have good stretchability. Nanocomposites can be more effective by utilizing the advantages of nanomaterials with different shapes and sizes. However, the cost of the materials increases, and it is difficult to handle and fabricate compared to a single material. The third method can append stretchability by using a structural design that has high stretchability, rather than using the advantages of nanomaterials. By introducing the structural design, this method has a greater possibility of increasing stretchability while having more flexibility in material selection. Wrinkle structures as well as sandwich, multilayer, auxetic, yarn, and porous structures are widely used. Normally, these structures are fine structures with nanometer to micrometer scale. Therefore, it is necessary to develop effective fabrication methods. The fabrication processes are discussed in detail in Sect. 4.4. In addition, studies on substrate 
(a)

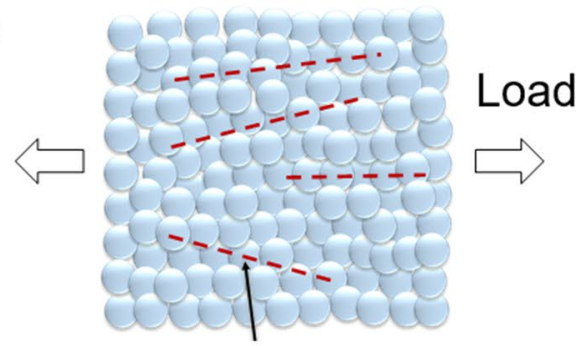

\section{Electron path}

(b)

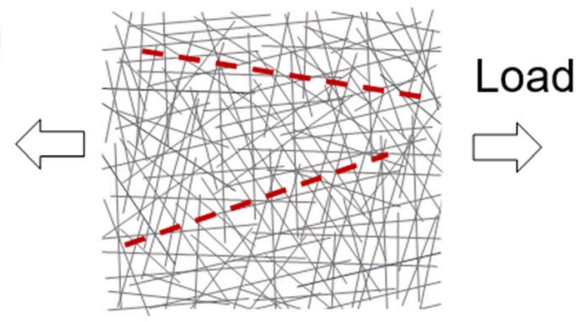

(c)

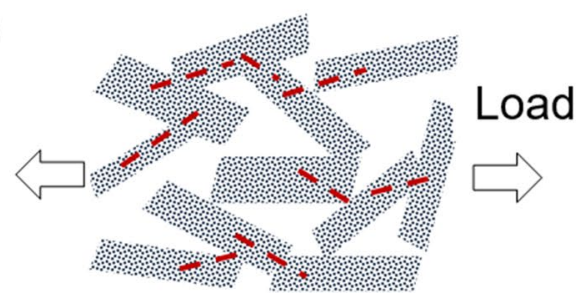

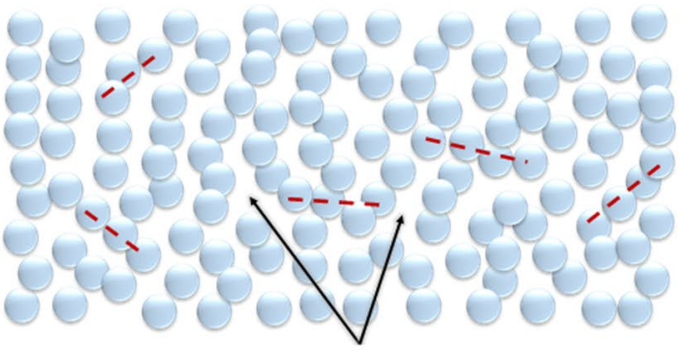

Opened cracks
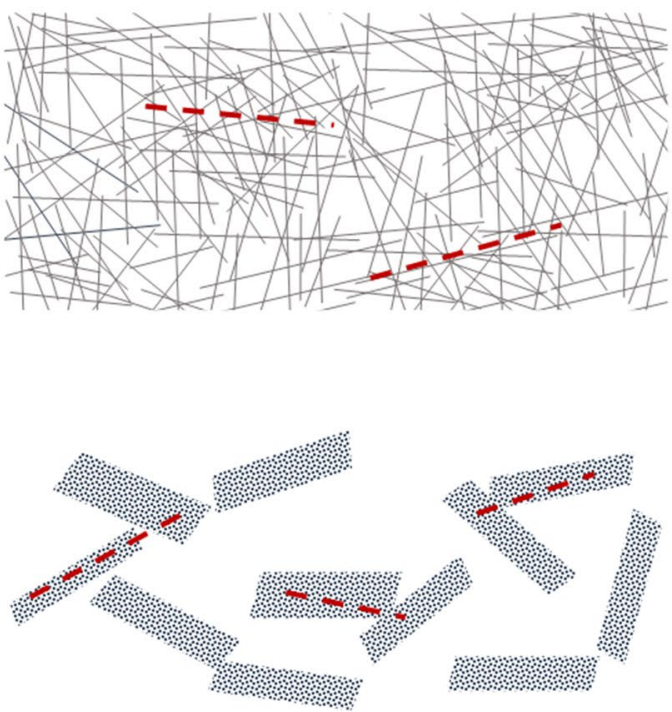

Fig. 18 The fabrication process of the (a) wrinkle structure, and (b) the yarn structure

materials that can stretch more than $1000 \%$ without cracks are required for a highly stretchable strain sensor.

\subsection{New Physics: Beyond the Limits of Wired Sensing}

There is a need for strain sensors that operate in environments that are hostile to humans. However, the requirement for additional data acquisition and the wire used to transmit sensor signals increase costs and may reduce accuracy. Thus, untethered (wireless) transmission capacity has been added to certain sensors. Such sensors can be used to monitor structural health when an electrical connection is expensive or when a human cannot enter the sensor environment (perhaps because of lethal gas). Wireless communication exploits various methods that are not mechanical or electrical (Table 5). Wireless communication is often achieved by integrating a commercial wireless communication module into a strain sensor [158, 200, 201]. However, only sensors with standalone wireless communication systems have been studied. RFID electromagnetic circuits are simple, cheap, and untethered. In addition, as the size of the material decreases, the magnetic characteristics become dominant. Therefore, a smaller magnetic field is required for magnetization. Cheng et al. developed a stretchable, largearea, wireless strain sensor using microfluidic, stretchable radiofrequency electronics. The sensor is fabricated as a mechanically reconfigurable antenna to measure the strain and communicate data via RF signals [84]. Kim et al. used silver nano-ink to create an antenna for LC resonator-based, chipless passive RFID tags [83]. The performance of wireless communications depends on the design of the patterns, normally in the range of hundreds of nanometers to tens of micrometers. In addition, to prevent any loss of space and make the product compact, it is important to design and print an antenna in a limited space. Therefore, direct printing methods are normally used to print nanomaterials and secure device density. Recently, strain sensors exploiting structural colors have been prepared via advanced optical nanofabrication technology. These sensors exploit Bragg's diffraction law. The distance between the nanostructures or fabricated nanopatterns varies within the wavelength range of visible light (380-700 $\mathrm{nm}$ ), and the strain changes the color. It should be noted that the GF unit for wireless 
(a)

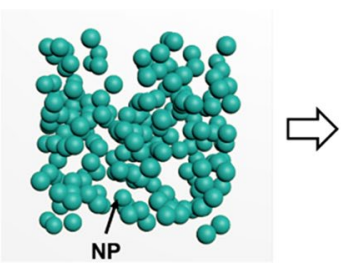

(b)

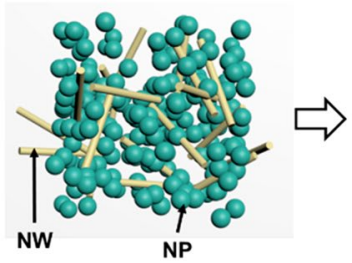

(c)

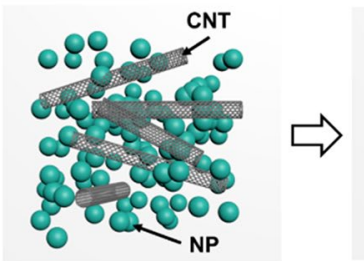

(d)

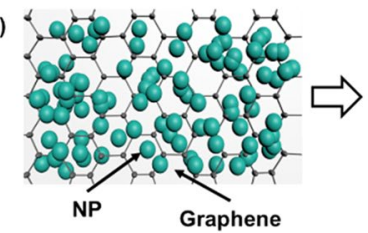

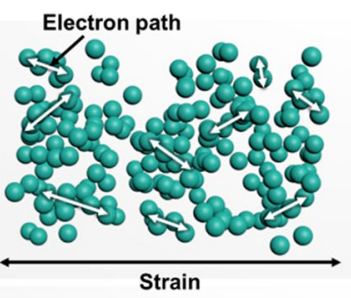

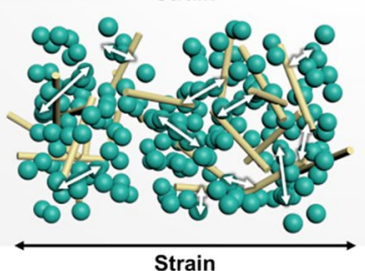

Strain
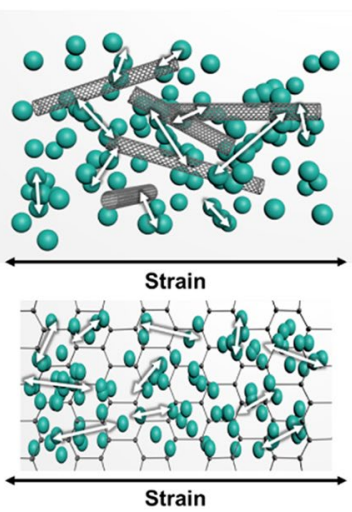

Fig. 19 A schematic of how stretchability may be imparted using nanocomposites composed of a nanoparticles alone and nanoparticles combined with $\mathbf{b}$ nanowires, $\mathbf{c}$ nanotubes, and $\mathbf{d}$ graphene

(a)

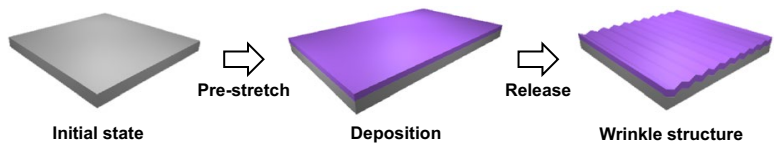

(b)

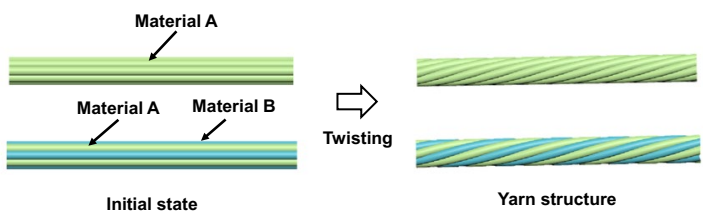

Fig. 20 A schematic of stretchability addition via mechanical cracking of a pre-stretched substrate

sensing employing optics (Table 5) is $\frac{\Delta \lambda}{\lambda} \varepsilon$. Most studies have used polymers. Park et al. employed a self-assembled block copolymer photonic crystal to develop a $100 \%$ stretchable, structural color strain sensor [192]. Fudouzi et al. embedded polystyrene colloidal particles in a PDMS film to change the structural color [193]. Shang et al. used an external magnetic

field to align superparamagnetic colloidal nanocrystal clusters into a 1D, chain-like, photonic crystal structure embedded in a polymer matrix [195]. Haque et al. employed unidirectionally aligned lamellar bilayers to develop a structural color in a hydrogel [197]. The hydrogel sheet can stretch by up to $60 \%$, and the color change is a measure of strain. $\mathrm{Wu}$ et al. developed a multi-responsive sensor using an oxidebased mechanoluminescent material. The sensor responds to both ultraviolet light and strain by changing its color [87]. Quan et al. developed a strain sensor using cone-shaped nanopatterns in a PDMS substrate; the sensor can measure large deformations (up to 100\%), biaxial 2D strain, and shear strain [86]. Thus, new physics facilitates the development of previously unimaginable applications; it does not just add new properties. Wireless communication technologies are gaining importance in the expanding wearable device market. The roles of nanomaterials and nanostructures increase accordingly.

\subsection{Combinations of Nanomaterials and Manufacturing Process}

Figures 21, 22, 23, 24 show the manufacturing processes combining nanoparticles, nanowires, nanotubes, and graphene, respectively. This illustrates a variety of real-world fabrication methods. Nanoparticle self-assembly was earlier a popular form of manufacturing (Fig. 21), but it has decreased over the past 10 years. Electrospinning is rapidly becoming popular. In addition, the demand for direct printing has increased. 3D printing is widely used for design autonomy and flexibility to mass-produce customized items and reflect each customer's needs. Chemical vapor deposition and physical vapor deposition processes have developed considerably and have reached their optimizations. They are frequently used in various nanomaterials. In particular, the chemical vapor deposition of nanowires and nanotubes has been widely studied. However, this method is currently less used to fabricate nanowires, but it remains commonly employed to create nanotubes. In addition, to mass-produce a variety of products and to meet a quick timeline of the products, molding has become popular. Research on the molding process is conducted to enable precise and rapid production, replacing conventional complex and expensive processes. Graphene structures are usually prepared via chemical vapor deposition and self-assembly, but 3D printing and molding are becoming more widely used. This analysis helps in understanding the trends and combination of the fabrication process used for handling various nanomaterials. This can be a guideline for researchers attempting to use nanomaterials for the first time.

The sensor market is expanding exponentially, and the importance of sensors is constantly increasing. Based on the literature, sensors made with nanomaterials exhibit high 


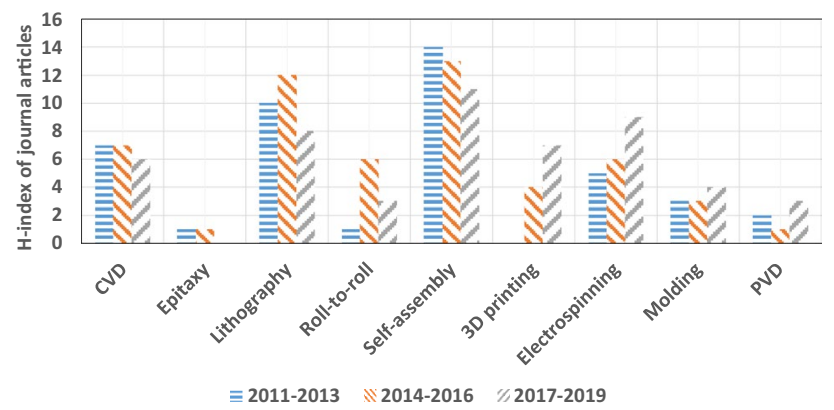

Fig. $21 \mathrm{H}$-index trajectory of keywords related to nanoparticles and their manufacture used between 2011 and 2013, 2014-2016, and 2017-2019. Data were obtained from the Web of Science in December 2019

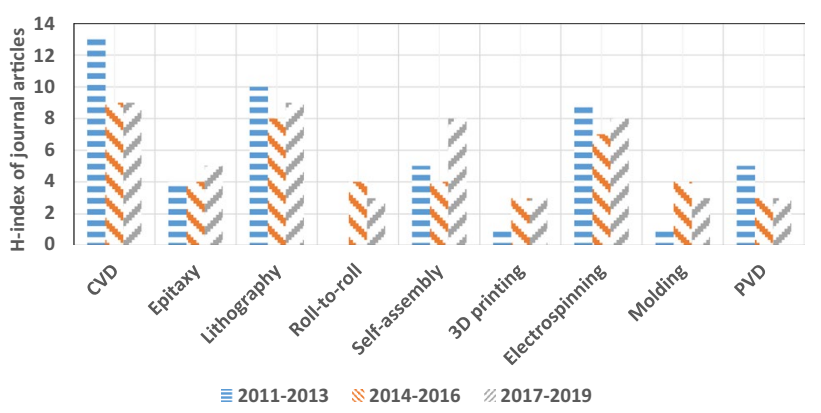

Fig. $22 \mathrm{H}$-index trajectory of keywords related to nanowires and the manufacture thereof used between 2011 and 2013, 2014-2016, and 2017-2019. Data were obtained from the Web of Science in December 2019

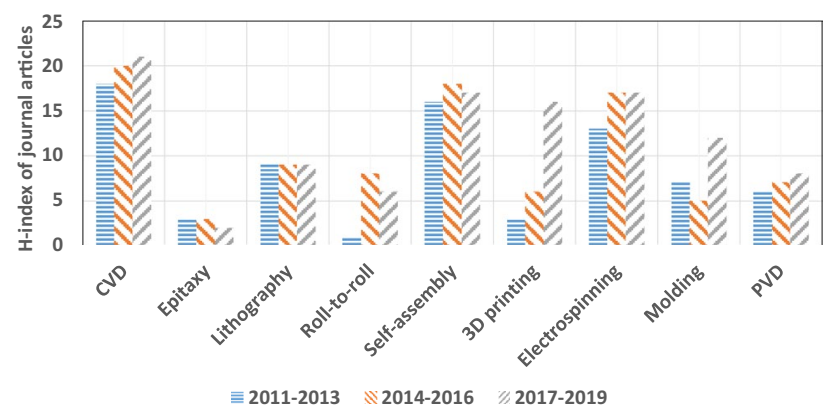

Fig. $23 \mathrm{H}$-index trajectory of keywords related to nanotubes and the manufacture thereof used between 2011 and 2013, 2014-2016, and 2017-2019. Data were obtained from the Web of Science in December 2019

performance and various functions. However, the cost of nanomaterials is high. The fabrication process and wireless sensing technologies still have considerable room for development. Engineers in the fields of mechanical, material, electrical, etc. need to collaborate to secure basic

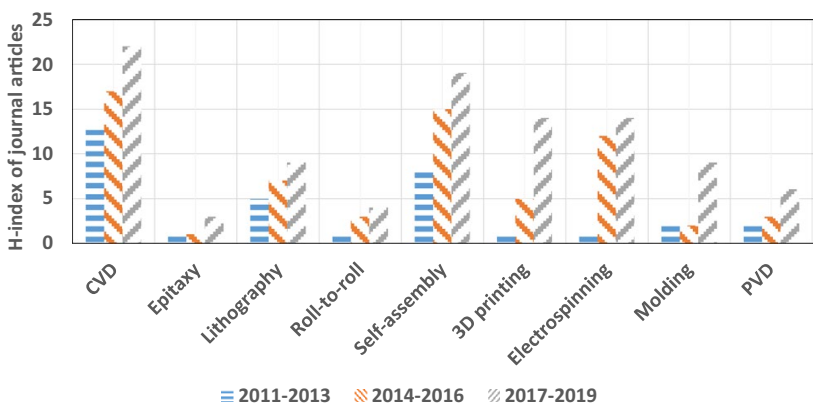

Fig. 24 H-index trajectory of keywords related to graphene and the manufacture thereof used between 2011 and 2013, 2014-2016, and 2017-2019. Data were obtained from the Web of Science in December 2019

technologies for the development and commercialization of sensors.

\section{Conclusions}

Nanomaterials have opened up new horizons in natural science and engineering. Scientists have focused on nanomaterial synthesis and physical phenomena that are lacking on a macro scale; furthermore, engineers are constantly seeking valuable applications. Multi-functionalization increases the value of nanomaterials by enhancing and appending properties and deriving new physics. We explored the use of nanomaterials in sensors, actuators, energy harvesters, and solar cells. We then applied our strategy to a sensor case study. We expect that not only sensors but also other devices will be multi-functionalized using novel nanomaterials. Our work will aid researchers in drawing research roadmaps that will enable them to achieve commercial success.

Acknowledgements This work was supported by the National Research Foundation of Korea (NRF) grant funded by the Korea government (MSIT) (NRF- 2021R1A4A2001824, 2018R1A2A13078704), and Institute of Engineering Research at Seoul National University.

\section{Declarations}

Conflict of interest On behalf of all authors, the corresponding author states that there is no conflict of interest.

Open Access This article is licensed under a Creative Commons Attribution 4.0 International License, which permits use, sharing, adaptation, distribution and reproduction in any medium or format, as long as you give appropriate credit to the original author(s) and the source, provide a link to the Creative Commons licence, and indicate if changes were made. The images or other third party material in this article are included in the article's Creative Commons licence, unless indicated otherwise in a credit line to the material. If material is not included in the article's Creative Commons licence and your intended use is not 
permitted by statutory regulation or exceeds the permitted use, you will need to obtain permission directly from the copyright holder. To view a copy of this licence, visit http://creativecommons.org/licenses/by/4.0/.

\section{References}

1. NNI 2014 Budget Supplement, National Nanotechnology Initiative. 2014.

2. 5 Coolest Things on Earth This Week, GE Reports. 2016.

3. Thompson, G. (2003). An intel perspective on silicon, nanotechnology and microelectronics. In: 1st Korea-US NanoForum, 2003.

4. Liu, Y., et al. (2015). Planar carbon nanotube-graphene hybrid films for high-performance broadband photodetectors. Nature Communications, 6(1), 1-7

5. Sun, D. M., et al. (2013). A review of carbon nanotube-and graphene-based flexible thin-film transistors. Small (Weinheim an der Bergstrasse, Germany), 9(8), 1188-1205

6. Byon, H. R., et al. (2013). Role of oxygen functional groups in carbon nanotube/graphene freestanding electrodes for high performance lithium batteries. Advanced Functional Materials, 23(8), 1037-1045

7. Zhu, G., et al. (2014). Highly conductive three-dimensional $\mathrm{MnO}_{2}$-carbon nanotube-graphene-Ni hybrid foam as a binderfree supercapacitor electrode. Nanoscale, 6(2), 1079-1085

8. Velten, J., et al. (2012). Carbon nanotube/graphene nanocomposite as efficient counter electrodes in dye-sensitized solar cells. Nanotechnology, 23(8), 085201

9. Gelperina, S., et al. (2005). The potential advantages of nanoparticle drug delivery systems in chemotherapy of tuberculosis. American Journal of Respiratory Critical Care Medicine, 172(12), 1487-1490

10. Mody, V. V., et al. (2014). Magnetic nanoparticle drug delivery systems for targeting tumor. Applied Nanoscience, 4(4), 385-392

11. Candini, A., et al. (2011). Graphene spintronic devices with molecular nanomagnets. Nano Letters, 11(7), 2634-2639

12. Dean, C. R., et al. (2010). Boron nitride substrates for high-quality graphene electronics. Nature Nanotechnology, 5(10), 722-726

13. Bonaccorso, F., et al. (2010). Graphene photonics and optoelectronics. Nature photonics, 4(9), 611

14. Das, S. K., et al. (2012). Self-assembled MoS2-carbon nanostructures: influence of nanostructuring and carbon on lithium battery performance. Journal of Materials Chemistry, 22(26), 12988-12992

15. Liu, M., Johnston, M. B., \& Snaith, H. J. (2013). Efficient planar heterojunction perovskite solar cells by vapour deposition. Nature, 501(7467), 395-398

16. Ding, Y., et al. (2007). A piezoelectric immunosensor for the detection of $\alpha$-fetoprotein using an interface of gold/hydroxyapatite hybrid nanomaterial. Biomaterials, 28(12), 2147-2154

17. Saif, M., Hafez, H., \& Nabeel, A. (2013). Photo-induced selfcleaning and sterilizing activity of $\mathrm{Sm} 3+$ doped $\mathrm{ZnO}$ nanomaterials. Chemosphere, 90(2), 840-847

18. Kalita, H., \& Karak, N. (2013). Bio-based hyperbranched polyurethane $/ \mathrm{Fe}_{3} \mathrm{O}_{4}$ nanocomposites as shape memory materials. Polymers for Advanced Technologies, 24(9), 819-823

19. Roy, S., Baral, A., \& Banerjee, A. (2013). An amino-acidbased self-healing hydrogel: modulation of the self-healing properties by incorporating carbon-based nanomaterials. Chemistry A European Journal, 19(44), 14950-14957

20. Maine, E., \& Seegopaul, P. (2016). Accelerating advancedmaterials commercialization. Nature Materials, 15(5), 487-491
21. Rafii-Tabar, H. (2000). Modelling the nano-scale phenomena in condensed matter physics via computer-based numerical simulations. Physics Reports, 325(6), 239-310

22. Kreyling, W. G., Semmler-Behnke, M., \& Chaudhry, Q. (2010). A complementary definition of nanomaterial. Nano Today, 5(3), $165-168$

23. Yun, Y.-H., et al. (2009). Tiny medicine: nanomaterial-based biosensors. Sensors, 9(11), 9275-9299

24. Mondal, S. K., et al. (2013). Evanescent wave assisted nanomaterial coating. Applied Optics, 52(22), 5455-5459

25. Srivastava, V. K., et al. (2017). Effect of nanomaterial on mode I and mode II interlaminar fracture toughness of woven carbon fabric reinforced polymer composites. Engineering Fracture Mechanics, 180, 73-86

26. Dosch, H. (2001). Some general aspects of confinement in nanomaterials. Applied Surface Science, 182(3-4), 192-195

27. Roduner, E. (2006). Size matters: why nanomaterials are different. Chemical Society Reviews, 35(7), 583-592

28. Lei, M., et al. (2009). Uncovering the intrinsic geometry from the atomic pair distribution function of nanomaterials. Physical Review B, 80(2), 024118

29. Lee, K. T., \& Cho, J. (2011). Roles of nanosize in lithium reactive nanomaterials for lithium ion batteries. Nano Today, 6(1), 28-41

30. Podyacheva, O. Y., \& Ismagilov, Z. (2015). Nitrogen-doped carbon nanomaterials: To the mechanism of growth, electrical conductivity and application in catalysis. Catalysis Today, 249, $12-22$

31. Zhang, J., et al. (2013). Improved hydrophilicity, permeability, antifouling and mechanical performance of PVDF composite ultrafiltration membranes tailored by oxidized low-dimensional carbon nanomaterials. Journal of Materials Chemistry A, 1(9), 3101-3111

32. Yu, X., \& Zhan, Z. (2014). The effects of the size of nanocrystalline materials on their thermodynamic and mechanical properties. Nanoscale Research Letters, 9(1), 516

33. Yao, J., Yang, M., \& Duan, Y. (2014). Chemistry, biology, and medicine of fluorescent nanomaterials and related systems: new insights into biosensing, bioimaging, genomics, diagnostics, and therapy. Chemical Reviews, 114(12), 6130-6178

34. Wang, J., et al. (2018). Synthesis, properties and applications of $\mathrm{ZnO}$ nanomaterials with oxygen vacancies: A review. Ceramics International, 44(7), 7357-7377

35. Alizada, A., Sofiyev, A., \& Kuruoglu, N. (2012). Stress analysis of a substrate coated by nanomaterials with vacancies subjected to uniform extension load. Acta Mechanica, 223(7), 1371-1383

36. Zhao, Y., et al. (2013). Nitrogen-doped carbon nanomaterials as non-metal electrocatalysts for water oxidation. Nature Communications, 4(1), 1-7

37. Jiang, C., \& Wang, Y. (2009). Formation of cobalt hollow nanospheres via surfactant-assisted hydrothermal progress. Materials Chemistry and Physics, 113(2-3), 531-533

38. Hong, R., et al. (2008). Facile route to $\gamma-\mathrm{Fe}_{2} \mathrm{O}_{3} / \mathrm{SiO}_{2}$ nanocomposite used as a precursor of magnetic fluid. Materials Chemistry and Physics, 108(1), 132-141

39. Lee, H.-T., et al. (2019). Research trends in sustainable manufacturing: A review and future perspective based on research databases. International Journal of Precision Engineering Manufacturing-Green Technology, 6, 1-11

40. Yoon, H.-S., et al. (2016). Future perspectives of sustainable manufacturing and applications based on research databases. International Journal of Precision Engineering Manufacturing, 17(9), 1249-1263

41. Clarivate Analytics, Web of Science. http://apps.webofknowl edge.com, Accessed DEC 2019, 2019.

42. Xiao, H., \& Liu, S. (2017). 2D nanomaterials as lubricant additive: A review. Materials \& Design, 135, 319-332 
43. Kaiser, J.-P., Zuin, S., \& Wick, P. (2013). Is nanotechnology revolutionizing the paint and lacquer industry? A critical opinion. Science of the Total Environment, 442, 282-289

44. Yang, X., et al. (2013). Model simulation and experiments of flow and mass transport through a nano-material gas filter. Applied Mathematical Modelling, 37(20-21), 9052-9062

45. Ren, H., et al. (2018). Thermal insulation characteristics of a lightweight, porous nanomaterial in high-temperature environments. Materials \& Design, 140, 376-386

46. Lin, J., Huang, Y., \& Huang, P. (2018). Graphene-based nanomaterials in bioimaging. Biomedical Applications of Functionalized Nanomaterials. (pp. 247-287). Elsevier.

47. Holzinger, M., Le Goff, A., \& Cosnier, S. (2014). Nanomaterials for biosensing applications: a review. Frontiers in Chemistry, 2, 63

48. Ji, T., et al. (2013). Using functional nanomaterials to target and regulate the tumor microenvironment: diagnostic and therapeutic applications. Advanced Materials, 25(26), 3508-3525

49. Bouwmeester, H., et al. (2014). State of the safety assessment and current use of nanomaterials in food and food production. Trends in Food Science \& Technology, 40(2), 200-210

50. Adams, W. J., \& Yellen, J. L. (1976). Commodity bundling and the burden of monopoly. The Quarterly Journal of Economics, 90, 475-498

51. Chuang, J.C.-I., \& Sirbu, M. A. (1999). Optimal bundling strategy for digital information goods: Network delivery of articles and subscriptions. Information Economics and Policy, 11(2), 147-176

52. Yadav, T. P., Yadav, R. M., \& Singh, D. P. (2012). Mechanical milling: a top down approach for the synthesis of nanomaterials and nanocomposites. Nanoscience and Nanotechnology, 2(3), 22-48

53. Wada, T., et al. (2014). Bulk-nanoporous-silicon negative electrode with extremely high cyclability for lithium-ion batteries prepared using a top-down process. Nano Letters, 14(8), $4505-4510$

54. Gregorczyk, K., \& Knez, M. (2016). Hybrid nanomaterials through molecular and atomic layer deposition: Top down, bottom up, and in-between approaches to new materials. Progress in Materials Science, 75, 1-37

55. Arole, V., \& Munde, S. (2014). Fabrication of nanomaterials by top-down and bottom-up approaches-an overview. Journal of Materials Science, 1, 89-93

56. Fischer, F., et al. (2008). On the role of surface energy and surface stress in phase-transforming nanoparticles. Progress in Materials Science, 53(3), 481-527

57. Bayender, B., et al. (1998). Solid-liquid surface energy of pivalic acid. Journal of Crystal Growth, 194(1), 119-124

58. Li, R., et al. (2016). Gibbs-Curie-Wulff theorem in organic materials: A case study on the relationship between surface energy and crystal growth. Advanced Materials, 28(8), $1697-1702$

59. Maine, E. (2008). Radical innovation through internal corporate venturing: Degussa's commercialization of nanomaterials. $R \& d$ Management, 38(4), 359-371

60. Arthur, D., et al. (2012). Carbon nanomaterial commercialization: Lessons for graphene from carbon nanotubes. MRS Bulletin, 37(12), 1297-1306

61. Borghetti, M., et al. (2016). Mechanical behavior of strain sensors based on PEDOT:PSS and silver nanoparticles inks deposited on polymer substrate by inkjet printing. Sensors and Actuators A: Physical, 243, 71-80

62. Agarwala, S., et al. (2017). Development of bendable strain sensor with embedded microchannels using 3D printing. Sensors and Actuators A: Physical, 263, 593-599
63. Zhao, J., Wang, G., Yang, R., Lu, X., Cheng, M., He, C., Xie, G., Meng, J., Shi, D., \& Zhang, G. (2015). Tunable piezoresistivity of nanographene films for strain sensing. ACS Nano, 9(2), $1622-1629$

64. Su, M., et al. (2016). Nanoparticle based curve arrays for multirecognition flexible electronics. Advanced Materials, 28(7), 1369-1374

65. Zhao, W., et al. (2015). Nanoparticle-structured highly sensitive and anisotropic gauge sensors. Small (Weinheim an der Bergstrasse, Germany), 11(35), 4509-4516

66. Zhang, S., et al. (2015). Highly stretchable, sensitive, and flexible strain sensors based on silver nanoparticles/carbon nanotubes composites. Journal of Alloys and Compounds, 652, 48-54

67. Park, J. J., et al. (2015). Highly stretchable and wearable graphene strain sensors with controllable sensitivity for human motion monitoring. ACS Applied Materials \& Interfaces, 7(11), $6317-6324$

68. Zhou, J., et al. (2017). Ultrasensitive, stretchable strain sensors based on fragmented carbon nanotube papers. ACS Applied Materials \& Interfaces, 9(5), 4835-4842

69. Roh, E., et al. (2015). Stretchable, transparent, ultrasensitive, and patchable strain sensor for human-machine interfaces comprising a nanohybrid of carbon nanotubes and conductive elastomers. ACS Nano, 9(6), 6252-6261

70. Liu, Q., et al. (2016). High-performance strain sensors with fish-scale-like graphene-sensing layers for full-range detection of human motions. ACS Nano, 10(8), 7901-7906

71. Gu, H., et al. (2019). Smart strain sensing organic-inorganic hybrid hydrogels with nano barium ferrite as the cross-linker. Journal of Materials Chemistry C, 7(8), 2353-2360

72. Guo, X., et al. (2017). Highly stretchable strain sensor based on SWCNTs/CB synergistic conductive network for wearable human-activity monitoring and recognition. Smart Materials and Structures, 26(9), 095017

73. Jeong, Y. R., et al. (2015). Highly stretchable and sensitive strain sensors using fragmentized graphene foam. Advanced Functional Materials, 25(27), 4228-4236

74. Matsuhisa, N., et al. (2017). Printable elastic conductors by in situ formation of silver nanoparticles from silver flakes. Nature Materials, 16(8), 834-840

75. Zhao, S., et al. (2017). Binary synergistic sensitivity strengthening of bioinspired hierarchical architectures based on fragmentized reduced graphene oxide sponge and silver nanoparticles for strain sensors and beyond. Small (Weinheim an der Bergstrasse, Germany), 13(28), 1700944

76. Jeon, J. Y., \& Ha, T. J. (2016). Waterproof electronic-bandage with tunable sensitivity for wearable strain sensors. ACS Applied Materials \& Interfaces, 8(4), 2866-2871

77. Wu, X., et al. (2016). Highly sensitive, stretchable, and washdurable strain sensor based on ultrathin conductive Layer@ Polyurethane yarn for tiny motion monitoring. ACS Applied Materials \& Interfaces, 8(15), 9936-9945

78. Liao, X., et al. (2017). Flexible, cuttable, and self-waterproof bending strain sensors using microcracked gold Nanofilms@ Paper substrate. ACS Applied Materials \& Interfaces, 9(4), 4151-4158

79. Shi, J., et al. (2016). Graphene reinforced carbon nanotube networks for wearable strain sensors. Advanced Functional Materials, 26(13), 2078-2084

80. Bae, S.-H., et al. (2013). Graphene-based transparent strain sensor. Carbon, 51, 236-242

81. Rahman, M. T., et al. (2018). 3D printed high performance strain sensors for high temperature applications. Journal of Applied Physics, 123(2), 024501 
82. Yi, L., et al. (2016). Ultrasensitive strain gauge with tunable temperature coefficient of resistivity. Nano Research, 9(5), 1346-1357

83. Kim, J., Wang, Z., \& Kim, W. S. (2014). Stretchable RFID for wireless strain sensing with silver nano ink. IEEE Sensors Journal, 14(12), 4395-4401

84. Cheng, S., \& Wu, Z. (2011). A microfluidic, reversibly stretchable, large-area wireless strain sensor. Advanced Functional Materials, 21(12), 2282-2290

85. Liu, C., et al. (2016). Tunable structural color surfaces with visually self-reporting wettability. Advanced Functional Materials, 26(43), 7937-7942

86. Quan, Y. J., et al. (2020). Stretchable biaxial and shear strain sensors using diffractive structural colors. ACS Nano, 14(5), 5392-5399

87. Wu, C., et al. (2018). Efficient mechanoluminescent elastomers for dual-responsive anticounterfeiting device and stretching/ strain sensor with multimode sensibility. Advanced Functional Materials, 28(34), 1803168

88. Snapp, P., et al. (2019). Colloidal photonic crystal strain sensor integrated with deformable graphene phototransducer. Advanced Functional Materials, 29(33), 1902216

89. Wang, H., et al. (2016). Downsized sheath-core conducting fibers for weavable superelastic wires, biosensors, supercapacitors, and strain sensors. Advanced Materials, 28(25), 4998-5007

90. Ferrreira, A., et al. (2012). Electromechanical performance of poly (vinylidene fluoride)/carbon nanotube composites for strain sensor applications. Sensors Actuators A: Physical, 178, 10-16

91. Pang, Y., et al. (2016). Flexible, highly sensitive, and wearable pressure and strain sensors with graphene porous network structure. ACS Applied Materials \& Interfaces, 8(40), 26458-26462

92. Liao, X., et al. (2016). A highly stretchable ZnO@ Fiber-based multifunctional nanosensor for strain/temperature/UV detection. Advanced Functional Materials, 26(18), 3074-3081

93. Lee, D., et al. (2016). Highly sensitive, transparent, and durable pressure sensors based on sea-urchin shaped metal nanoparticles. Advanced Materials, 28(42), 9364-9369

94. Liu, X., et al. (2017). A highly sensitive graphene woven fabric strain sensor for wearable wireless musical instruments. Materials Horizons, 4(3), 477-486

95. Yan, T., et al. (2018). Carbon/graphene composite nanofiber yarns for highly sensitive strain sensors. Materials \& Design, 143, 214-223

96. Chen, S., et al. (2016). Ultrasensitive cracking-assisted strain sensors based on silver nanowires/graphene hybrid particles. ACS Applied Materials \& Interfaces, 8(38), 25563-25570

97. Shen, T., et al. (2017). Remotely triggered locomotion of hydrogel mag-bots in confined spaces. Science and Reports, 7(1), 16178

98. Watanabe, S., Era, H., \& Kunitake, M. (2018). Two-wavelength infrared responsive hydrogel actuators containing rare-earth photothermal conversion particles. Science and Reports, 8(1), 13528

99. Gladman, A. S., et al. (2016). Biomimetic 4D printing. Nature Materials, 15(4), 413-418

100. Liu, L., et al. (2016). Giving direction to motion and surface with ultra-fast speed using oriented hydrogel fibers. Advanced Functional Materials, 26(7), 1021-1027

101. Yang, C., et al. (2017). Reduced graphene oxide-containing smart hydrogels with excellent electro-response and mechanical properties for soft actuators. ACS Applied Materials \& Interfaces, 9(18), 15758-15767

102. Kim, Y. S., et al. (2015). Thermoresponsive actuation enabled by permittivity switching in an electrostatically anisotropic hydrogel. Nature Materials, 14(10), 1002-1007
103. Sun, Z., Yamauchi, Y., Araoka, F., Kim, Y. S., Bergueiro, J., Ishida, Y., Ebina, Y., Sasaki, T., Hikima, T., \& Aida, T. (2018). An anisotropic hydrogel actuator enabling earthworm-like directed peristaltic crawling. Angewandte Chemie, 130(48), 15998-16002

104. Zhu, S. E., et al. (2011). Graphene-based bimorph microactuators. Nano Letters, 11(3), 977-981

105. Han, L., et al. (2018). Multiresponsive actuators based on modified electrospun films. RSC Advances, 8(19), 10302-10309

106. Shepherd, H. J., et al. (2013). Molecular actuators driven by cooperative spin-state switching. Nature Communications, 4 2607

107. Terasawa, N., \& Asaka, K. (2018). High-performance graphene oxide/vapor-grown carbon fiber composite polymer actuator. Sensors and Actuators B: Chemical, 255, 2829-2837

108. Kim, S.-S., et al. (2015). High-fidelity bioelectronic muscular actuator based on graphene-mediated and TEMPO-oxidized bacterial cellulose. Advanced Functional Materials, 25(23), 3560-3570

109. Li, H., et al. (2016). Magnetic actuated pH-responsive hydrogelbased soft micro-robot for targeted drug delivery. Smart Materials and Structures, 25(2), 027001

110. Wang, K., Stenner, C., \& Weissmüller, J. (2017). A nanoporous gold-polypyrrole hybrid nanomaterial for actuation. Sensors and Actuators B: Chemical, 248, 622-629

111. Ge, F., et al. (2017). An optical actuator based on gold-nanoparticle-containing temperature-memory semicrystalline polymers. Angewandte Chemie (International ed. in English), 56(22), $6126-6130$

112. Zheng, L., et al. (2019). Dual-stimulus smart actuator and robot hand based on a vapor-responsive PDMS film and triboelectric nanogenerator. ACS Applied Materials \& Interfaces, 11(45), 42504-42511

113. Shin, S. R., et al. (2015). Aligned carbon nanotube-based flexible gel substrates for engineering bio-hybrid tissue actuators. Advanced Functional Materials, 25(28), 4486-4495

114. Lu, S., \& Panchapakesan, B. (2006). Nanotube micro-optomechanical actuators. Applied Physics Letters, 88(25), 253107

115. Wang, E., Desai, M. S., \& Lee, S. W. (2013). Light-controlled graphene-elastin composite hydrogel actuators. Nano Letters, 13(6), 2826-2830

116. Xiao, P., et al. (2016). Construction of a fish-like robot based on high performance graphene/PVDF bimorph actuation materials. Adv Sci (Weinh), 3(6), 1500438

117. Liang, J., et al. (2011). Electromechanical actuators based on graphene and graphene $/ \mathrm{Fe}_{3} \mathrm{O}_{4}$ hybrid paper. Advanced Functional Materials, 21(19), 3778-3784

118. Park, S., et al. (2010). Graphene-based actuators. Small (Weinheim an der Bergstrasse, Germany), 6(2), 210-212

119. Li, W., et al. (2016). Flexible circuits and soft actuators by printing assembly of graphene. ACS Applied Materials \& Interfaces, 8(19), 12369-12376

120. Sul, O., \& Yang, E. H. (2009). A multi-walled carbon nanotube-aluminum bimorph nanoactuator. Nanotechnology, 20(9), 095502

121. Kong, L., \& Chen, W. (2014). Carbon nanotube and graphenebased bioinspired electrochemical actuators. Advanced Materials, 26(7), 1025-1043

122. Deng, J., et al. (2016). Tunable photothermal actuators based on a pre-programmed aligned nanostructure. Journal of the American Chemical Society, 138(1), 225-230

123. Jeong, C. K., et al. (2015). A hyper-stretchable elastic-composite energy harvester. Advanced Materials, 27(18), 2866-2875

124. Shi, K., et al. (2018). Synergistic effect of graphene nanosheet and $\mathrm{BaTiO}_{3}$ nanoparticles on performance enhancement of 
electrospun PVDF nanofiber mat for flexible piezoelectric nanogenerators. Nano Energy, 52, 153-162

125. Liu, K., et al. (2018). Piezoelectric energy harvesting and charging performance of $\mathrm{Pb}(\mathrm{Zn} 1 / 3 \mathrm{Nb} 2 / 3) \mathrm{O} 3-\mathrm{Pb}(\mathrm{Zr} 0.5 \mathrm{Ti} 0.5) \mathrm{O} 3$ nanoparticle-embedded P(VDF-TrFE) nanofiber composite sheets. Composites Science and Technology, 168, 296-302

126. Shin, D.-J., et al. (2019). Enhanced flexible piezoelectric energy harvesters based on $\mathrm{BaZrTiO}_{3}-\mathrm{BaCaTiO}_{3}$ nanoparticles/PVDF composite films with $\mathrm{Cu}$ floating electrodes. Journal of Alloys and Compounds, 802, 562-572

127. Zhang, Y., et al. (2018). Flexible energy harvesting polymer composites based on biofibril-templated 3-dimensional interconnected piezoceramics. Nano Energy, 50, 35-42

128. Rahman, W., et al. (2018). Self-powered piezoelectric nanogenerator based on wurtzite $\mathrm{ZnO}$ nanoparticles for energy harvesting application. Materials Today: Proceedings, 5(3), 9826-9830

129. Alam, M. M., Sultana, A., \& Mandal, D. (2018). Biomechanical and acoustic energy harvesting from $\mathrm{TiO}_{2}$ nanoparticle modulated PVDF Nanofiber made high performance nanogenerator. ACS Applied Energy Materials, 1(7), 3103-3112

130. Dutta, B., et al. (2018). NiO@SiO2/PVDF: a flexible polymer nanocomposite for a high performance human body motionbased energy harvester and tactile e-skin mechanosensor. ACS Sustainable Chemistry \& Engineering, 6(8), 10505-10516

131. Baek, C., et al. (2016). A flexible energy harvester based on a lead-free and piezoelectric BCTZ nanoparticle-polymer composite. Nanoscale, 8(40), 17632-17638

132. Ding, R., et al. (2016). Flexible piezoelectric nanocomposite generators based on formamidinium lead halide perovskite nanoparticles. Advanced Functional Materials, 26(42), 7708-7716

133. Wang, L., Yang, X., \& Daoud, W. A. (2019). High power-output mechanical energy harvester based on flexible and transparent Au nanoparticle-embedded polymer matrix. Nano Energy, 55, $433-440$

134. Kar, E., et al. (2019). 2D SnO2 nanosheet/PVDF composite based flexible, self-cleaning piezoelectric energy harvester. Energy Conversion and Management, 184, 600-608

135. Baek, C., et al. (2018). Enhanced output performance of a leadfree nanocomposite generator using $\mathrm{BaTiO}_{3}$ nanoparticles and nanowires filler. Applied Surface Science, 429, 164-170

136. Gupta, M. K., Kim, S. W., \& Kumar, B. (2016). Flexible highperformance lead-free $\mathrm{Na} 0.47 \mathrm{~K} 0.47 \mathrm{Li} 0.06 \mathrm{NbO}_{3}$ microcubestructure-based piezoelectric energy harvester. ACS Applied Materials Interfaces, 8(3), 1766-1773

137. Ren, X., et al. (2016). Flexible lead-free $\mathrm{BiFeO}_{3} / \mathrm{PDMS}$-based nanogenerator as piezoelectric energy harvester. ACS Applied Materials \& Interfaces, 8(39), 26190-26197

138. Chen, J., et al. (2016). Enhancing performance of triboelectric nanogenerator by filling high dielectric nanoparticles into sponge PDMS film. ACS Applied Materials \& Interfaces, 8(1), 736-744

139. Zhu, J., Zhu, Y., \& Wang, X. (2018). A hybrid piezoelectric and triboelectric nanogenerator with PVDF nanoparticles and leaf-shaped microstructure ptfe film for scavenging mechanical energy. Advanced Materials Interfaces, 5(2), 1700750

140. Kim, D., Jin, I. K., \& Choi, Y. K. (2018). Ferromagnetic nanoparticle-embedded hybrid nanogenerator for harvesting omnidirectional vibration energy. Nanoscale, 10(26), 12276-12283

141. Zi, W., et al. (2016). Ag nanoparticle enhanced light trapping in hydrogenated amorphous silicon germanium solar cells on flexible stainless steel substrate. Solar Energy Materials and Solar Cells, 144, 63-67

142. Bush, K. A., et al. (2016). Thermal and environmental stability of semi-transparent perovskite solar cells for tandems enabled by a solution-processed nanoparticle buffer layer and sputtered ITO electrode. Advanced Materials, 28(20), 3937-3943
143. Dou, Y., et al. (2016). Enhanced performance of dye-sensitized solar cell using Bi2Te3 nanotube/ZnO nanoparticle composite photoanode by the synergistic effect of photovoltaic and thermoelectric conversion. Journal of Power Sources, 307, 181-189

144. Deka, N., et al. (2018). Enhancing solar cell efficiency with plasmonic behavior of double metal nanoparticle system. Vacuum, 152, 285-290

145. Lee, K.-W., et al. (2016). Enhanced photovoltaic performance of back-illuminated dye-sensitized solar cell based on $\mathrm{TiO}_{2}$ nanoparticle/nanowire composite film in cobalt redox system. Journal of Alloys and Compounds, 656, 568-572

146. Xu, Y., et al. (2017). Ag-encapsulated single-crystalline anatase $\mathrm{TiO}_{2}$ Nanoparticle photoanodes for enhanced dye-sensitized solar cell performance. Journal of Alloys and Compounds, 695, $1104-1111$

147. Xu, L., et al. (2017). Stable monolithic hole-conductor-free perovskite solar cells using TiO 2 nanoparticle binding carbon films. Organic Electronics, 45, 131-138

148. Liu, X., et al. (2016). Au nanoparticle enhanced thin-film silicon solar cells. Solar Energy Materials and Solar Cells, 147, $225-234$

149. Wang, W.-T., et al. (2018). Nanoparticle-induced fast nucleation of pinhole-free $\mathrm{PbI} 2$ film for ambient-processed highly-efficient perovskite solar cell. Nano Energy, 49, 109-116

150. Wang, W., et al. (2016). A $4.92 \%$ efficiency $\mathrm{Cu}_{2} \mathrm{ZnSnS}_{4}$ solar cell from nanoparticle ink and molecular solution. RSC Advances, 6(59), 54049-54053

151. Miskin, C. K., et al. (2015). $9.0 \%$ efficient $\mathrm{Cu}_{2} \mathrm{ZnSn}(\mathrm{S}, \mathrm{Se})_{4}$ solar cells from selenized nanoparticle inks. Progress in Photovoltaics: Research and Applications, 23(5), 654-659

152. McLeod, S. M., et al. (2015). Synthesis and characterization of $15 \%$ efficient CIGSSe solar cells from nanoparticle inks. Progress in Photovoltaics: Research and Applications, 23(11), $1550-1556$

153. Wang, Y., et al. (2017). The light-induced field-effect solar cell concept—perovskite nanoparticle coating introduces polarization enhancing silicon cell efficiency. Advanced Materials, 29(18), 1606370

154. Ma, J., et al. (2017). MgO nanoparticle modified anode for highly efficient $\mathrm{SnO}_{2}$-based planar perovskite solar cells. Advance Science (Weinh), 4(9), 1700031

155. Zang, Y., et al. (2015). Advances of flexible pressure sensors toward artificial intelligence and health care applications. Materials Horizons, 2(2), 140-156

156. Lee, J., et al. (2014). A stretchable strain sensor based on a metal nanoparticle thin film for human motion detection. Nanoscale, 6(20), 11932-11939

157. Puyoo, E., et al. (2017). Metallic nanoparticle-based strain sensors elaborated by atomic layer deposition. J Applied Physics Letters, 110(12), 123103

158. Amjadi, M., et al. (2014). Highly stretchable and sensitive strain sensor based on silver nanowire-elastomer nanocomposite. ACS Nano, 8(5), 5154-5163

159. Wei, Y., et al. (2016). Multiscale wrinkled microstructures for piezoresistive fibers. Advanced Functional Materials, 26(28), 5078-5085

160. Kim, K. K., et al. (2015). Highly sensitive and stretchable multidimensional strain sensor with prestrained anisotropic metal nanowire percolation networks. Nano Letters, 15(8), 5240-5247

161. $\mathrm{Hu}, \mathrm{B}$., et al. (2010). External-strain induced insulating phase transition in $\mathrm{VO}_{2}$ nanobeam and its application as flexible strain sensor. Advanced Materials, 22(45), 5134-5139

162. Pan, S., et al. (2019). Mechanocombinatorially screening sensitivity of stretchable strain sensors. Advanced Materials, 31(35), 1903130 
163. Yu, X.-G., et al. (2017). A wearable strain sensor based on a carbonized nano-sponge/silicone composite for human motion detection. Nanoscale, 9(20), 6680-6685

164. Zhang, M., et al. (2017). Carbonized cotton fabric for high-performance wearable strain sensors. Advanced Functional Materials, 27(2), 1604795

165. Wang, C., et al. (2016). Carbonized silk fabric for ultrastretchable, highly sensitive, and wearable strain sensors. Advanced Materials, 28(31), 6640-6648

166. Yamada, T., et al. (2011). A stretchable carbon nanotube strain sensor for human-motion detection. Nature Nanotechnology, 6(5), 296

167. Li, C., et al. (2015). Flexible CNT-array double helices strain sensor with high stretchability for motion capture. Scientific Reports, 5(1), 1-8

168. Park, S. J., et al. (2016). Highly flexible wrinkled carbon nanotube thin film strain sensor to monitor human movement. Advanced Materials Technologies, 1(5), 1600053

169. Foroughi, J., et al. (2016). Knitted carbon-nanotube-sheath/ spandex-core elastomeric yarns for artificial muscles and strain sensing. ACS Nano, 10(10), 9129-9135

170. Ryu, S., et al. (2015). Extremely elastic wearable carbon nanotube fiber strain sensor for monitoring of human motion. ACS Nano, 9(6), 5929-5936

171. Zhao, H., et al. (2010). Carbon nanotube yarn strain sensors. Nanotechnology, 21(30), 305502

172. Liu, H., et al. (2016). Electrically conductive strain sensing polyurethane nanocomposites with synergistic carbon nanotubes and graphene bifillers. Nanoscale, 8(26), 12977-12989

173. Yin, G., et al. (2011). A carbon nanotube/polymer strain sensor with linear and anti-symmetric piezoresistivity. Journal of composite materials, 45(12), 1315-1323

174. Christ, J. F., et al. (2017). 3D printed highly elastic strain sensors of multiwalled carbon nanotube/thermoplastic polyurethane nanocomposites. Materials Design, 131, 394-401

175. Liu, Z., et al. (2015). Thickness-gradient films for high gauge factor stretchable strain sensors. Advanced Materials, 27(40), 6230-6237

176. Wang, Z., et al. (2016). Polyurethane/cotton/carbon nanotubes core-spun yarn as high reliability stretchable strain sensor for human motion detection. ACS Applied Materials \& Interfaces, 8(37), 24837-24843

177. Jiang, Y., et al. (2018). Auxetic mechanical metamaterials to enhance sensitivity of stretchable strain sensors. Advanced Materials, 30(12), 1706589

178. Cheng, Y., et al. (2015). A stretchable and highly sensitive graphene-based fiber for sensing tensile strain, bending, and torsion. Advanced Materials, 27(45), 7365-7371

179. Li, J., et al. (2016). Highly stretchable and sensitive strain sensor based on facilely prepared three-dimensional graphene foam composite. ACS Applied Materials Interfaces, 8(29), 18954-18961

180. Li, X., et al. (2016). Large-area ultrathin graphene films by single-step marangoni self-assembly for highly sensitive strain sensing application. Advanced Functional Materials, 26(9), $1322-1329$

181. Li, X., et al. (2012). Stretchable and highly sensitive grapheneon-polymer strain sensors. Scientific Reports, 2, 870

182. Rahimi, R., et al. (2015). Highly stretchable and sensitive unidirectional strain sensor via laser carbonization. ACS Applied Materials Interfaces, 7(8), 4463-4470

183. Liu, Q., et al. (2015). High-quality graphene ribbons prepared from graphene oxide hydrogels and their application for strain sensors. ACS Nano, 9(12), 12320-12326

184. Tang, Y., et al. (2015). Highly stretchable and ultrasensitive strain sensor based on reduced graphene oxide microtubes-elastomer composite. ACS Applied Materials \& Interfaces, 7(49), 27432-27439

185. Wang, Y., et al. (2018). Flexible electrically resistive-type strain sensors based on reduced graphene oxide-decorated electrospun polymer fibrous mats for human motion monitoring. Carbon, $126,360-371$

186. Shi, G., et al. (2016). Highly sensitive, wearable, durable strain sensors and stretchable conductors using graphene/silicon rubber composites. Advanced Functional Materials, 26(42), 7614-7625

187. Liao, X., et al. (2015). Flexible and highly sensitive strain sensors fabricated by pencil drawn for wearable monitor. Advanced Functional Materials, 25(16), 2395-2401

188. Liao, X., et al. (2016). Flexible and printable paper-based strain sensors for wearable and large-area green electronics. Nanoscale, $8(26), 13025-13032$

189. Li, M., et al. (2014). Stretchable conductive polypyrrole/polyurethane $(\mathrm{PPy} / \mathrm{PU})$ strain sensor with netlike microcracks for human breath detection. ACS Applied Materials Interfaces, 6(2), 1313-1319

190. Chossat, J.-B., et al. (2013). A soft strain sensor based on ionic and metal liquids. IEEE Sensors Journal, 13(9), 3405-3414

191. Guo, J., et al. (2016). Highly stretchable, strain sensing hydrogel optical fibers. Advanced Materials, 28(46), 10244-10249

192. Park, T. H., et al. (2018). Block copolymer structural color strain sensor. NPG Asia Materials, 10(4), 328-339

193. Fudouzi, H., \& Sawada, T. (2006). Photonic rubber sheets with tunable color by elastic deformation. Langmuir, 22(3), $1365-1368$

194. Ito, T., et al. (2013). Strain-responsive structural colored elastomers by fixing colloidal crystal assembly. Langmuir, 29(45), 13951-13957

195. Shang, S., et al. (2015). Facile fabrication of a magnetically induced structurally colored fiber and its strain-responsive properties. Journal of Materials Chemistry A, 3(20), 11093-11097

196. Chan, E. P., et al. (2011). Block copolymer photonic gel for mechanochromic sensing. Advanced Materials, 23(40), 4702-4706

197. Haque, M. A., et al. (2010). Unidirectional alignment of lamellar bilayer in hydrogel: one-dimensional swelling, anisotropic modulus, and stress/strain tunable structural color. Advanced Materials, 22(45), 5110-5114

198. Sandt, J. D., et al. (2018). Stretchable optomechanical fiber sensors for pressure determination in compressive medical textiles. Advance Healthcare Materials, 7(15), e1800293

199. Minati, L., et al. (2017). Gold nanoparticles 1D array as mechanochromic strain sensor. Materials Chemistry and Physics, 192, 94-99

200. Eom, J., et al. (2017). Highly sensitive textile strain sensors and wireless user-interface devices using all-polymeric conducting fibers. ACS Applied Materials \& Interfaces., 9(11), 10190-10197

201. Lee, T., et al. (2016). Flexible textile strain wireless sensor functionalized with hybrid carbon nanomaterials supported $\mathrm{ZnO}$ nanowires with controlled aspect ratio. Advanced Functional Materials, 26(34), 6206-6214

Publisher's Note Springer Nature remains neutral with regard to jurisdictional claims in published maps and institutional affiliations. 


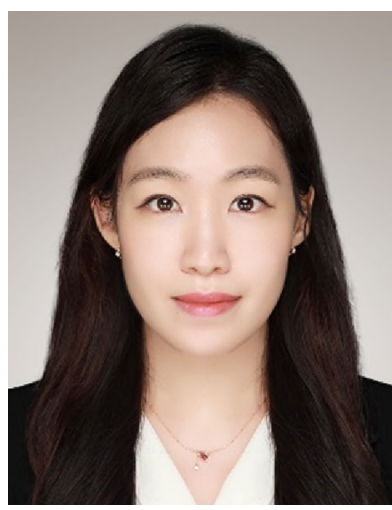

Ji-Hyeon Song received a joint $\mathrm{Ph} . \mathrm{D}$. degree in mechanical engineering from Seoul National University and Georgia Institute of Technology in 2019. She is currently an assistant professor at Dankook University. Her research interests include micro-/ nanoscale fabrication and multiscale computational simulation.

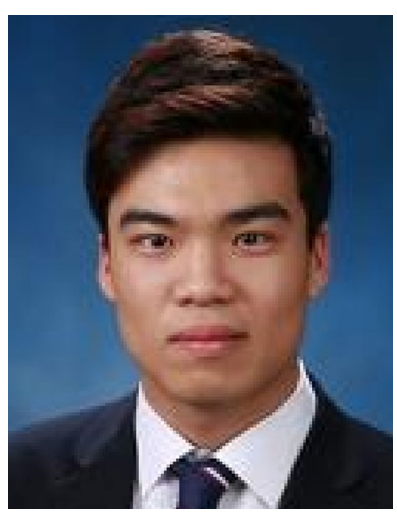

Soo-Hong Min received his Ph.D. degree in mechanical engineering from Seoul National University in 2020 . He is currently working in Mechatronics R\&D Center, Samsung Electronics. His research interests include micro-/nanoscale fabrication, flexible and printed sensor, and smart manufacturing system.

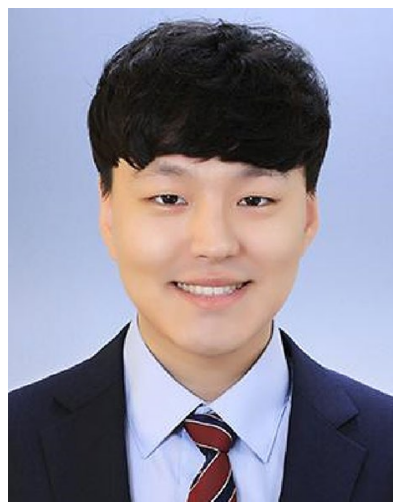

Seung-Gi Kim received an MS degree in mechanical engineering from Seoul National University in 2021 . He is currently a researcher in Samsung Display. His research interests include micro/nanoscale fabrication, smart manufacturing system, and AIoT.

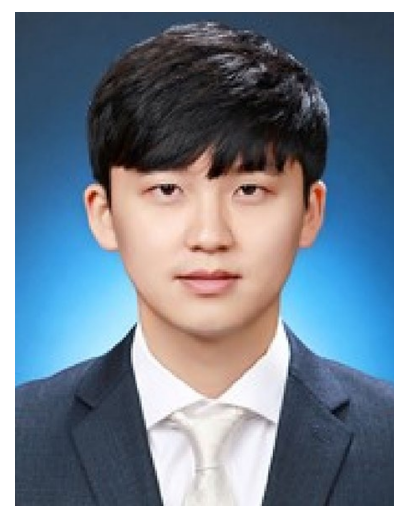

Younggyun Cho received an MS degree in mechanical engineering from Seoul National University in 2021 . He is currently a researcher in Global Technology Center at Samsung Electronics. His research interests include micro/nanoscale fabrication, structural color sensor, and smart manufacturing system.

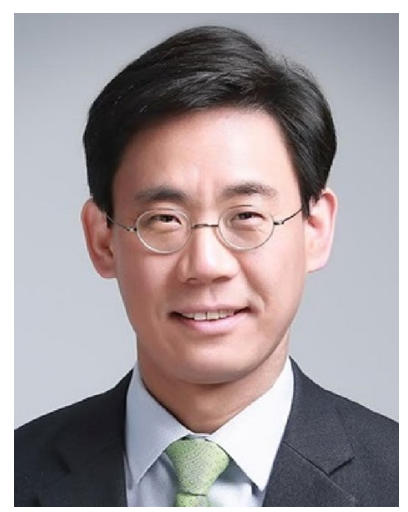

Sung-Hoon Ahn received a Ph.D. degree from Stanford University in 1997. He has been affiliated with UC Berkeley, Gyeongsang National University, University of Washington, and Hyundai WIA Corp. He is currently a full professor in the department of mechanical and aerospace engineering at Seoul National University. His research interests include smart factory, green manufacturing, soft robotics, 3D printing, composite materials, micro/nano fabrications, renewtechnology. able energy, and appropriate 